TOPICAL REVIEW

\section{Fundamentals of the 3-3-1 model with heavy leptons}

To cite this article: F C Correia 2018 J. Phys. G: Nucl. Part. Phys. 45043001

View the article online for updates and enhancements.

\section{Related content}

The HigasTools handbook: a beginners

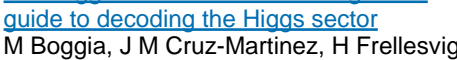
et al.

- Heavy neutral pseudoscalar decays into gauge bosons in the Littlest Higqs model J I Aranda, E Cruz-Albaro, D EspinosaGómez et al.

Lectures on Yangian symmetry Florian Loebbert

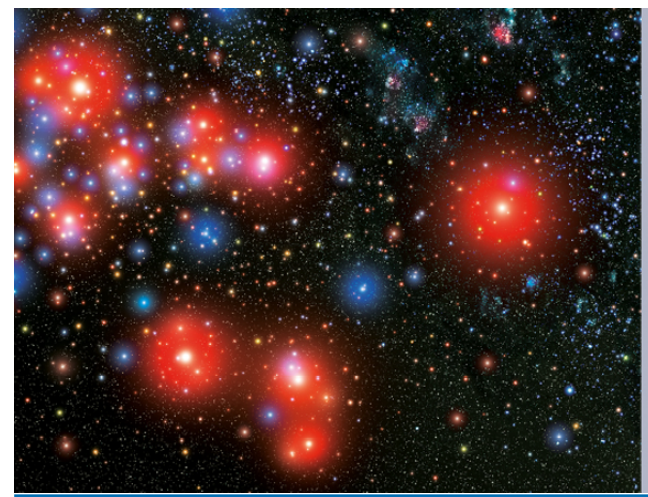

A.A IOP Astronomy ebooks

Part of your publishing universe and your first choice for astronomy, astrophysics, solar physics and planetary science ebooks. iopscience.org/books/aas 


\title{
Fundamentals of the 3-3-1 model with heavy leptons
}

\author{
F C Correia ${ }^{1,2}$ (1) \\ ${ }^{1}$ Institute for Theoretical Physics, Sao Paulo State University-UNESP. Sao Paulo, SP \\ 01140070, Brazil \\ ${ }^{2}$ Instituto de Física Corpuscular, Universitat de Valéncia. E-46980 Paterna, Spain \\ E-mail: ccorreia@ift.unesp.br
}

Received 14 May 2017, revised 17 November 2017

Accepted for publication 31 January 2018

Published 19 February 2018

\begin{abstract}
This work is a brief presentation of the theory based on the $S U(3)_{c} \otimes$ $S U(3)_{L} \otimes U(1)_{X}$ gauge group in the presence of heavy leptons. Recent studies [1] have considered a set of four possible variants for the 3-3-1HL, whose content arises according to the so-denoted variable $\beta$. Since it has been argued about the presence of stable charged particles in this sort of model, we divide the different sectors of the Lagrangian between universal and specific vertices, and conclude that the omission of $\beta$-dependent terms in the potential may induce discrete symmetry for the versions defined by $|\beta|=\sqrt{3}$. In the context of $|\beta|=\frac{1}{\sqrt{3}}$, where the new degrees of freedom have the same standard electric charges, additional Yukawa interactions may create decay channels into the SM sector. Furthermore, motivated by a general consequence of the Goldstone theorem, a method of diagonalization by parts is introduced in the Scalar sector and provides a clarification on the definition of mass eigenstates. In summary, we develop the most complete set of terms allowed by the symmetry group and resolve their definitive pieces in order to justify the model description present in the literature.
\end{abstract}

Keywords: 3-3-1 models, heavy leptons, heavy quarks

\section{Introduction}

The new gauge-structure defining the electroweak sector of 3-3-1 models [2, 3], namely $S U(3)_{L} \otimes U(1)_{X}$, can render important phenomenological consequences and has been the object of attention in recent years. Among some recent analysis, it can be mentioned those 
based on the context of collider [1] and low-energy physics [4-9], WIMPs [10, 11] and different possible extensions [12].

The model has a total of six versions ${ }^{3}$, two of which filling the lepton content with a conjugate of standard particles, thus defining the minimal 3-3-1 (see [14] and references therein). The present work is a brief review of the variant including new heavy leptons [15-18], here denoted as 3-3-1HL. It will be shown that a different particle content may be defined according to a discrete variable $\beta$ limited to a set of four possible values $[1,19]$. The total Lagrangian, divided into boson and fermion sectors, is then explored by first introducing the totality of $\beta$-independent terms. At this point we aim to conclude about the presence of stable charged particles, as stated in [1]. These features arise whenever only a universal potential is considered, i.e. when the scalar self-interactions are assumed to be defined exclusively from generic terms present in all versions of the model. The omission of $\beta$ specific interactions will retain the mixing from the potential following the same pattern as that present in the gauge-fixing Lagrangian. Next, the complete case is developed and a method that explores our previous knowledge on the gauge-dependent sector is introduced in order to simplify the search for the mass eigenstates.

This review comprises the first part of a work in progress that intends to apply a general integration method for these sort of models, resulting in an effective theory that might be directly tested through precision observables. Thus, our first task involves the development of a consistent notation followed by a systematic classification of the interactions among new and standard terms. We ultimately must select and treat those pieces that can generate 6-dimension operators at tree and loop-level. Apart from that, one exclusive assumption is consistently considered along our analysis-the first breaking scale must be much larger than the second one, with the notation translated into $u \gg v_{\rho}, v_{\eta}$.

Finally, the same steps of the authors in [20] along their presentation of the Standard Model are freely followed. Like any gauge theory with spontaneous symmetry breaking, the total Lagrangian is composed of the gauge-kinetic interactions of scalars and fermions, self-interactions of bosons and a Yukawa, such that the review can be organized as follows:

- Section 2 : Gauge structure and scalars in the 3-3-1HL

- 2.1: The particle content in different versions;

-2.2 : Self-interactions of scalars;

-2.3 : Vacuum stability condition;

- 2.4 : Gauge-fixing Lagrangian;

-2.5 : Gauge-boson masses;

- 2.6 : Scalar masses;

- 2.7 : The potential for particular models;

-2.8 : Self-interactions of gauge bosons;

- Section 3 : Fermions in the 3-3-1HL

- 3.1: Gauge interactions of the fermions;

- 3.2 : Yukawa Lagrangian;

- Section 4 Conclusions.

${ }^{3}$ In addition, there is a variant where the heavy leptons present fractional electric charges [13]. These versions will not be treated here. 


\section{Gauge structure and scalars in the $3-3-1 \mathrm{HL}$}

The root of electroweak interactions is expressed by the structure of the covariant derivative. In the context of a $S U(3)_{L} \otimes U(1)_{X}$ gauge group it can be represented as:

$$
D_{\mu}=\partial_{\mu}+\mathrm{i} g \mathbf{W}_{\mu} \cdot \mathbf{I}+\mathrm{i} g_{X} X W_{\mu}^{0} \mathbb{I}
$$

where the bold letter express a simple vector $\mathbf{A} \equiv\left(A^{1}, A^{2}, \cdots, A^{8}\right)$ and $I^{a}=\frac{\lambda^{a}}{2}$ are the $S U(3)$ generators. By expanding the gauge piece of $D_{\mu}$ explicitly we can divide it into complex (or non-diagonal) and real interactions

$$
D_{\mu}^{(C C)}=\mathrm{i} \frac{g}{2}\left(\begin{array}{ccc}
0 & W_{\mu}^{1}-\mathrm{i} W_{\mu}^{2} & W_{\mu}^{4}-\mathrm{i} W_{\mu}^{5} \\
W_{\mu}^{1}+\mathrm{i} W_{\mu}^{2} & 0 & W_{\mu}^{6}-\mathrm{i} W_{\mu}^{7} \\
W_{\mu}^{4}+\mathrm{i} W_{\mu}^{5} & W_{\mu}^{6}+\mathrm{i} W_{\mu}^{7} & 0
\end{array}\right)
$$

and

$$
D_{\mu}^{(N C)}=\mathrm{i}\left(\begin{array}{ccc}
\frac{g}{2}\left(W_{\mu}^{3}+\frac{W_{\mu}^{8}}{\sqrt{3}}\right)+g_{X} X W_{\mu}^{0} & 0 & 0 \\
0 & \frac{g}{2}\left(-W_{\mu}^{3}+\frac{W_{\mu}^{8}}{\sqrt{3}}\right)+g_{X} X W_{\mu}^{0} & 0 \\
0 & 0 & -\frac{g}{\sqrt{3}} W_{\mu}^{8}+g_{X} X W_{\mu}^{0}
\end{array}\right)
$$

The assertion that the fields presented in equation $(2.2 a)$ will be associated with charged currents would be the first sign on how we will construct both the particle content of the theory and the pattern of symmetry breaking. Nevertheless, as is shown in section 2.1, there are in effect two different variants of the model where neutral currents may also reside in nondiagonal vertices.

The following representation to the fermionic fields is going to be considered:

- Leptons $\psi_{\alpha}:\left(\begin{array}{ll}\left(\nu_{\alpha} l_{\alpha}\right) & E_{\alpha}\end{array}\right)_{L}^{\top},\left(\mathbf{1}, \mathbf{3}, \mathrm{X}_{\psi}\right)$

- Quarks $Q_{i}:\left(\left(d_{i} u_{i}\right) J_{i}\right)_{L}^{\top},\left(\mathbf{3}, \overline{3}, X_{Q}\right)$

- Quarks $Q_{3}:\left(\begin{array}{lll}\left(u_{3} d_{3}\right) & J_{3}\end{array}\right)_{L}^{\top},\left(\mathbf{3}, \mathbf{3}, X_{3}\right)$

- $u_{a}^{R}:\left(3,1, \frac{2}{3}\right)$

- $d_{a}^{R}:\left(\mathbf{3}, \mathbf{1},-\frac{1}{3}\right)$

- $l_{\alpha}^{R}:(\mathbf{1}, \mathbf{1},-1)$

- $J_{i}^{R}:\left(\mathbf{3}, \mathbf{1}, X_{J}\right)$

- $\mathrm{J}_{3}^{R}:\left(3,1, X_{J_{3}}\right)$

- $E_{\alpha}^{R}:\left(1,1, X_{E}\right)$,

where $\alpha=[e, \mu, \tau], a=[1,2,3]$ and $i=[1,2]$. The brackets inside the triplets denote that these are in reality doublets of $S U(2)$ or, implicitly, that the gauge group will follow a hierarchy under the symmetry breaking such that, in one of its steps, these objects compose a new symmetric Lagrangian comprising the Standard Model. Apart from that, we omit some of the hypercharges and leave their derivation to the following subsection.

The above mentioned hierarchy is endowing the model with a new scale represented by the letter $u$ and present for the breaking of $S U(3)_{L} \otimes U(1)_{X}$ into $S U(2)_{L} \otimes U(1)_{Y}$. The scale 
is introduced via the vacuum expectation value of a new neutral scalar $\chi^{0}$ singlet of $S U(2)$, composing the triplet

$$
\chi=\left(\begin{array}{lll}
\left(\begin{array}{ll}
\chi^{V} & \chi^{U}
\end{array}\right) & \chi^{0}
\end{array}\right)^{\top}
$$

Through the next sections we will discuss that the 3-3-1HL must also include two additional scalar triplets, such that it might also appear in an intermediate scale via Higgs interactions. Nevertheless, for a matter of clarity and driven by a current phenomenology that disallow new degrees of freedom to show up at low energies, we may perform our task of separating the model into standard and new exotic sectors by considering only the first breaking. In other words, we can place the classical electroweak symmetry breaking aside, enclosed in a $\mathcal{L}_{\mathrm{SM}}$ function. Thus, our primary focus is on an Universe ruled by nine interactions, where five of them are mediated by massive and four by massless vector particles. The former acquire their masses when $\chi^{0}$ presents a vacuum expectation value (vev) $\left\langle\chi^{0}\right\rangle=u$, or $\langle\chi\rangle \propto\left(\begin{array}{lll}0 & 0 & u\end{array}\right)^{\top}$, leading the theory to $\mathcal{L}_{331} \rightarrow \mathcal{L}_{\mathrm{SM}}+\mathrm{NP}$.

Inside the set of nine $S U(3)_{L} \otimes U(1)_{X}$ generators, the following four leave the vacuum unbroken and may define a basis for the group $S U(2)_{L} \otimes U(1)_{Y}[5]$

$$
\mathbb{T}_{1}\langle\chi\rangle=\mathbb{T}_{2}\langle\chi\rangle=\mathbb{T}_{3}\langle\chi\rangle=\left(\beta \mathbb{T}_{8}+X \mathbb{I}\right)\langle\chi\rangle=0
$$

From our knowledge of SM symmetry breaking, in general we can extract a connection between electromagnetism and weak interactions represented by the so called Gell-MannNishijima relation

$$
\mathbb{Q}=\mathbb{T}_{3}+\frac{\mathbb{Y}}{2}
$$

where, in our case,

$$
\frac{\mathbb{Y}}{2}=\beta \mathbb{T}_{8}+X \mathbb{I}
$$

i.e. a diagonal generator for the SM symmetry group defining the particles hypercharge and the respective conserved currents.

Equation (2.4b) introduces a new and decisive variable. The parameter $\beta$ may completely alter the model phenomenology and is an element of a set limited to four numbers, namely $\left\{ \pm \sqrt{3}, \pm \frac{1}{\sqrt{3}}\right\}$. As presented in [1], this set is generated by factors which includes the integer nature of the electric charge for asymptotic states and the positiveness for variables of mass squared. For illustration, this section is concluded with the demonstration of some results and identities in the context of $\beta=-\sqrt{3}$ (or $X_{\chi}=-1$ ). This version contains a single charged heavy lepton and the previous relations may provide us, for example, the charges of exotic quarks inside the triplets.

Since the quarks $J$ are singlet of $S U(2)$, it follows

$$
\begin{aligned}
& Q_{3}=\left(\begin{array}{l}
u_{3} \\
d_{3} \\
J_{3}
\end{array}\right) \quad \rightarrow \quad \mathbb{Q}_{Q_{3}}=\left(\begin{array}{ccc}
\frac{2}{3} & & \\
& -\frac{1}{3} & \\
& & q_{J_{3}}
\end{array}\right) \\
& \therefore \quad \frac{2}{3}=\left(\mathbb{T}_{3}\right)_{11}-\sqrt{3}\left(\mathbb{T}_{8}\right)_{11}+X_{3} \\
& \quad=X_{3} .
\end{aligned}
$$


The $J_{3}$ electric charge will then be given by

$$
q_{J_{3}}=-\sqrt{3}\left(\mathbb{T}_{8}\right)_{33}+\frac{2}{3}=\frac{5}{3} .
$$

The gauge boson $\left(W^{4}-\mathrm{i} W^{5}\right)$, for instance, will couple with the current

$$
j_{(u J)}^{\mu} \equiv \bar{u}_{3 L} \gamma^{\mu} J_{3 L}
$$

thus corresponding to a particle charged by $(-1)$. Similarly,

$$
j_{(d J)}^{\mu} \equiv \bar{d}_{3 L} \gamma^{\mu} J_{3 L}
$$

coupled to the so-denoted U-boson and whose charge, in this case, must be equal to $(-2)$.

By considering the three $S U(2)$ subalgebras of $S U(3)$ defined by the raising and lowering operators:

$$
\begin{array}{ll}
\mathbb{I}_{ \pm}=\frac{\mathbb{T}_{1} \pm \mathrm{i} \mathbb{T}_{2}}{\sqrt{2}}, & \mathbb{I}_{3}=\mathbb{T}_{3} \\
\mathbb{J}_{ \pm}=\frac{\mathbb{T}_{4} \pm \mathrm{i} \mathbb{T}_{5}}{\sqrt{2}}, & \mathbb{J}_{3}=\frac{\sqrt{3}}{2} \mathbb{T}_{8}-\frac{\mathbb{T}_{3}}{2} \\
\mathbb{L}_{ \pm}=\frac{\mathbb{T}_{6} \pm \mathrm{i} \mathbb{T}_{7}}{\sqrt{2}}, & \mathbb{L}_{3}=\frac{\sqrt{3}}{2} \mathbb{T}_{8}+\frac{\mathbb{T}_{3}}{2}
\end{array}
$$

and also the gauge bosons (note the changes on the signs for $V$ and $U$ compared with $W$ ):

$$
W^{ \pm}=\frac{W^{1} \mp \mathrm{i} W^{2}}{\sqrt{2}}, \quad V^{ \pm}=\frac{W^{4} \pm \mathrm{i} W^{5}}{\sqrt{2}}, \quad U^{ \pm \pm}=\frac{W^{6} \pm \mathrm{i} W^{7}}{\sqrt{2}}
$$

the charged sector of $D_{\mu}$ can be expressed in a short notation as

$D_{\mu}^{(C C)}=\mathrm{i} g\left(W^{+} \mathbb{I}_{+}+W^{-} \mathbb{I}_{-}+V^{-} \mathbb{J}_{+}+V^{+} \mathbb{J}_{-}+U^{--} \mathbb{L}_{+}+U^{++} \mathbb{L}_{-}\right)$.

In order to obtain the interactions in the conjugate representation we just change the raising and lowering operators sign:

$D_{\mu}^{*(C C)}=-\mathrm{i} g\left(W^{+} \mathbb{I}_{-}+W^{-} \mathbb{I}_{+}+V^{-} \mathbb{J}_{-}+V^{+} \mathbb{J}_{+}+U^{--} \mathbb{L}_{-}+U^{++} \mathbb{L}_{+}\right)$.

It is common to present the quark representations with a minus sign in the definition of $Q_{i}$, in general, aiming to recover the exact aspect of the SM Lagrangian. Nevertheless, such phase insertion does not have any phenomenological implications.

- Note: To find the Gell-Mann-Nishijima relation to the fields in conjugate representation we must remember that the conjugation of the generators $\mathbb{T}_{3}$ and $\mathbb{T}_{8}$ is defined as

$$
\mathbb{T}_{3}^{*}=-\mathbb{T}_{3}, \quad \mathbb{T}_{8}^{*}=-\mathbb{T}_{8}
$$

and, therefore,

$$
\mathbb{Q}=-\mathbb{T}_{3}+\sqrt{3} \mathbb{T}_{8}+X \mathbb{I} .
$$

Let us find, for example, $X_{Q}$ and $q_{J_{i}}$ : 


$$
\begin{aligned}
\mathbb{Q}_{Q_{i}}=\left(\begin{array}{ccc}
-\frac{1}{3} & & \\
& \frac{2}{3} & \\
& & q_{J_{i}}
\end{array}\right) \rightarrow \frac{2}{3} & =\left(-\mathbb{T}_{3}\right)_{22}+\sqrt{3}\left(\mathbb{T}_{8}\right)_{22}+X_{Q} \\
& =\frac{1}{2}+\frac{1}{2}+X_{Q} .
\end{aligned}
$$

Thus,

$$
\therefore \quad X_{Q}=-\frac{1}{3} \quad \rightarrow \quad q_{J_{i}}=\sqrt{3}\left(\mathbb{T}_{8}\right)_{33}-\frac{1}{3}=-\frac{4}{3} .
$$

Now we move to the diagonal terms of the covariant derivative, those corresponding to the neutral current interactions:

$$
D_{\mu}^{(N C)}=\mathrm{i} g\left(\mathbb{T}_{3} W^{3}+\mathbb{T}_{8} W^{8}\right)+\mathrm{i} g_{X} X \llbracket W^{0} .
$$

After the first breaking by $\langle\chi\rangle, W^{0}$ will mix with $W^{8}$ and can be rotated into the mass eigenstates by

$$
\left(\begin{array}{l}
W^{0} \\
W^{8}
\end{array}\right)=\left(\begin{array}{cc}
c_{x} & -s_{x} \\
s_{x} & c_{x}
\end{array}\right)\left(\begin{array}{c}
B \\
Z^{\prime}
\end{array}\right)
$$

which implies

$$
\begin{aligned}
D_{\mu}^{(N C)} & =\mathrm{i} g \mathbb{T}_{3} W^{3}+\mathrm{i} g \mathbb{T}_{8}\left(B s_{x}+Z^{\prime} c_{x}\right)+\mathrm{i} g_{X} X \mathbb{I}\left(B c_{x}-Z^{\prime} s_{x}\right) \\
& =\mathrm{i}\left(\mathbf{g}_{W^{3}} W^{3}+\mathbf{g}_{B} B+\mathbf{g}_{Z^{\prime}} Z^{\prime}\right)
\end{aligned}
$$

where it has been defined

$$
\mathbf{g}_{W^{3}} \equiv g \mathbb{T}_{3}, \quad \mathbf{g}_{B} \equiv g \mathbb{T}_{8} s_{x}+g_{X} X \mathbb{I}_{x}, \quad \mathbf{g}_{Z^{\prime}} \equiv \mathbb{T}_{8} c_{x}-g_{X} X \mathbb{I}_{s_{x}}
$$

The mixing between $B$ and $W^{3}$ after the second symmetry breaking can also be anticipated without loss of generality

$$
\left(\begin{array}{c}
B \\
W^{3}
\end{array}\right)=\left(\begin{array}{cc}
c_{w} & -s_{w} \\
s_{w} & c_{w}
\end{array}\right)\left(\begin{array}{l}
A \\
Z
\end{array}\right)
$$

or, finally,

$$
D_{\mu}^{(N C)}=\mathrm{i}\left(\mathbf{g}_{A} A+\mathbf{g}_{Z} Z+\mathbf{g}_{Z^{\prime}} Z^{\prime}\right)
$$

where,

$$
\mathbf{g}_{A} \equiv s_{w} \mathbf{g}_{W^{3}}+c_{w} \mathbf{g}_{B}, \quad \mathbf{g}_{Z} \equiv c_{w} \mathbf{g}_{W^{3}}-s_{w} \mathbf{g}_{B} .
$$

In order to require that electromagnetic interactions be reproduced, we must impose

$$
\mathbf{g}_{A}=e \mathbb{Q}
$$

or

$$
e \mathbb{Q}=g \mathbb{T}_{3} s_{w}+g \mathbb{T}_{8} s_{x} c_{w}+g_{X} X \mathbb{I} c_{x} c_{w} .
$$

Equation (2.25), when applied for different quarks and leptons, may result in some important relations to the coupling constants. For instance, to the quark- $d_{1}$ $\left(q_{d}=-\frac{1}{3}, X_{Q}=-\frac{1}{3}\right)$ we have: 


$$
-\frac{e}{3}=-\left(\frac{g}{2} s_{w}+\frac{g}{2 \sqrt{3}} s_{x} c_{w}\right)-\frac{g_{X}}{3} c_{x} c_{w} .
$$

From equation (2.28) and by assuming $e=g s_{w}$ :

$$
-\frac{e}{3}=-\left(\frac{e}{2}-\frac{e}{2}\right)-\frac{g_{X}}{3} c_{x} c_{w} \quad \rightarrow \quad g_{X} c_{x} c_{w}=e
$$

or, finally, if $g^{\prime}=\frac{e}{c_{w}}$,

$$
g_{X} c_{x}=g^{\prime}
$$

which connects the hypercharge sectors of the Standard Model and the 3-3-1HL. Besides, from the results of the next section, derived for a generic version of the model, it can be shown that equation (2.27) is in fact $\beta$ independent. We must note, however, that the same relations raised in the context of the Standard Model were considered, what is to assert equivalent angles and couplings.

If we repeat our use of equation (2.25) to the case of neutrinos $\left(q_{\nu}=0, X_{\psi}=0\right)$ we find

$\frac{g}{2} s_{w}+\frac{g}{2 \sqrt{3}} s_{x} c_{w}=0 \quad \rightarrow \quad s_{x}=-\sqrt{3} \tan \theta_{w} \quad\left(\right.$ or $\left.s_{x}=\beta \tan \theta_{w}\right)$

i.e. a connection between the Weinberg angle and the mixing angle from the first breaking. The general formula inside the brackets can also be proved from the identities in the next section and provides a direct manner to count the possible variants for the 3-3-1HL. Once $|\beta|=\frac{n}{\sqrt{3}}$, the condition $|\beta|<\frac{1}{\tan \theta_{w}}$ will force $n \in[1,2,3]$. The models with $n=2$ present fractional electric charges both for the heavy leptons and the new gauge bosons (see equations (2.33) and (2.38)). This variant has been considered in [13] and will not be treated here. Moreover, those variants where $n=3$ leave just a small window to the renormalization group evolution of $\mathrm{s}_{w}^{2}$. According to [21], the Weinberg angle can reach $\tan ^{2} \theta_{w}=\frac{1}{3}$ at $M_{Z^{\prime}} \sim 4 \mathrm{TeV}$, leading $g_{X}$ in equation (2.27) to a Landau pole. Therefore, models with $|\beta|=\sqrt{3}$ are already very constrained by unitary bounds [13]. In section (2.5) is derived a set of natural relations for the coupling constants and the gauge boson masses which may convert this assertion into a similar conclusion on the $Z^{\prime}$ mass. On what follows we aim to be general in presenting the totality of $\beta$-dependent results and select particular examples for $|\beta|=\sqrt{3}$ in order to connect the review with the works $[2,3,15]$.

The rotation of $\left(B, W^{3}\right)$ into $(A, Z)$ is in reality neglecting the mixing with $Z^{\prime}$ emerged after the second breaking. This assumption is also motivated by the large difference between the scales, $u \gg v$. The equations (2.28) and (2.27) induce a set of simplified expressions for the $Z$ and $Z^{\prime}$ couplings. For instance,

$$
\begin{aligned}
& \mathbf{g}_{Z}=g \mathbb{T}_{3} c_{w}-\left(g \mathbb{T}_{8} s_{x}+g_{X} X \mathbb{I} c_{x}\right) s_{w} \\
& \stackrel{(2.27)}{=} g \mathbb{T}_{3} c_{w}-\left(g \mathbb{T}_{8} s_{X}+g^{\prime} X \mathbb{I}\right) s_{w} \\
& \stackrel{(2.28)}{=} g c_{w} \mathbb{T}_{3}-g \tan \theta_{w} s_{w}\left(\beta \mathbb{T}_{8}+X \mathbb{I}\right) \\
& =g c_{w} \mathbb{T}_{3}+g \frac{s_{w}^{2}}{c_{w}}\left(\mathbb{T}_{3}-\mathbb{Q}\right) \\
& =\frac{g}{c_{w}}\left(\mathbb{T}_{3}-\mathbb{Q} s_{w}^{2}\right)
\end{aligned}
$$

i.e. the well-known coupling of $Z$ with the SM particles. The fourth equal sign consider $g^{\prime}=g \tan \theta_{w}$ and the result is $\beta$-independent. A similar expression can also be achieved for $Z^{\prime}$ through 


$$
\begin{aligned}
\mathbf{g}_{Z^{\prime}} & =g \mathbb{T}_{8} c_{x}-g_{X} X \mathbb{I} s_{x} \\
\stackrel{(2.27)}{=} & g\left(\mathbb{T}_{8} c_{x}-\tan \theta_{w} \tan \theta_{x} X \mathbb{I}\right) \\
\stackrel{(2.28)}{=} & \frac{g}{c_{x}}\left(\mathbb{T}_{8} c_{x}^{2}-\frac{s_{x}^{2}}{\beta} X \mathbb{I}\right) .
\end{aligned}
$$

The relation (2.29) also exhibits the massless nature of $Z$ after the first breaking

$$
\frac{g}{c_{w}}\left(\left(\mathbb{T}_{3}\right)_{33}-0 * s_{w}^{2}\right)=0
$$

i.e. there is no coupling with the neutral scalar in $\chi$.

Thus, the section may be concluded with the total covariant derivative expressed under a simplified notation,

$$
\begin{aligned}
D_{\mu}= & \mathbb{I} \partial_{\mu}+\mathrm{i}\left[g\left(W^{+} \mathbb{I}_{+}+W^{-} \mathbb{I}_{-}\right)+\mathbf{g}_{Z} Z+e \mathbb{Q} A\right] \\
& +\mathrm{i}\left[g\left(V^{-} \mathbb{J}_{+}+V^{+} \mathbb{J}_{-}+U^{--} \mathbb{L}_{+}+U^{++} \mathbb{L}_{-}\right)+\mathbf{g}_{Z^{\prime}} Z^{\prime}\right] .
\end{aligned}
$$

The first line reproduces exactly the Standard Model contribution and will make simpler the task of dividing the 3-3-1HL into SM and new physics elements.

As mentioned previously, the present work intends to specify how the different sectors of the Lagrangian will connect the new degrees of freedom with the standard fields at tree- and loop-level. In order to be consistent and provide an independent review, we remake in detail some of the main aspects of the model, being however substantially supported by previous works like [1,2] and [5]. When the context allows, we persist regarding the first breaking $S U(3)_{L} \otimes U(1)_{X} \rightarrow S U(2)_{L} \otimes U(1)_{Y}$ with priority. In the next section some identities for a general 3-3-1HL version are extracted.

\subsection{Particle content in different versions}

First, let us recall the electric charge operator:

$$
\mathbb{Q}=\mathbb{T}_{3}+\beta \mathbb{T}_{8}+X \mathbb{I} .
$$

Then we start to find the electric charges dependent on $\beta$. From the first line of $D_{\mu} \chi$, for example, it follows $q_{\chi_{1}}=1+q_{\chi_{2}}=q_{V}$, which can also justify why the first entry of $\chi$ has been defined as $\chi_{V}$. Besides, the second line would give us $q_{U}=q_{\chi_{2}}$, and we denote $\chi_{2} \equiv \chi_{U}$.

The neutral third component of $\chi$ implies $X_{\chi}=\frac{\beta}{\sqrt{3}}$, i.e.

$$
\begin{aligned}
q_{\chi_{V}}=q_{V} & =\frac{1}{2}+\frac{\beta}{2 \sqrt{3}}+\frac{\beta}{\sqrt{3}} \\
& =\frac{\sqrt{3}}{2} \beta+\frac{1}{2} \quad \rightarrow \quad q_{U}=\frac{\sqrt{3}}{2} \beta-\frac{1}{2} .
\end{aligned}
$$

By repeating this simple procedure to the quarks and leptons representations we obtain their respective hypercharges. For example, the value of $X_{Q}$, defined in the conjugated representation, will be given by

$$
X_{Q}=\frac{1}{6}+\beta \frac{1}{2 \sqrt{3}} \quad \rightarrow \quad q_{j_{i}}=\frac{1}{6}+\frac{\sqrt{3}}{2} \beta
$$


or, for the triplet in the fundamental representation,

$$
X_{3}=\frac{1}{6}-\frac{\beta}{2 \sqrt{3}} \rightarrow q_{j_{3}}=\frac{1}{6}-\frac{\sqrt{3}}{2} \beta .
$$

As mentioned before, the electric charges of the new gauge bosons, as well as the $Z^{\prime}$ mass, prevent $\beta$ of assuming any possible value [1], restricting it to the set

$$
\beta \in\left[ \pm \frac{1}{\sqrt{3}}, \pm \sqrt{3}\right]
$$

From equations (2.34) and (2.35) it is clear the effect of a \pm sign for quarks is merely of inverting the electric charges into different representations. For $|\beta|=\sqrt{3}$ the possible values are $q_{J} \in\left[-\frac{4}{3}, \frac{5}{3}\right]$ and for $|\beta|=\frac{1}{\sqrt{3}}, q_{J} \in\left[-\frac{1}{3}, \frac{2}{3}\right]$.

Similarly, for the leptons we have:

$$
\psi_{\alpha}:\left(\begin{array}{c}
\nu_{\alpha} \\
l_{\alpha} \\
E_{\alpha}
\end{array}\right)_{L} \quad \rightarrow \quad X_{\psi}=-\left(\frac{1}{2}+\frac{\beta}{2 \sqrt{3}}\right)
$$

or

$$
q_{E}=-\left(\frac{1}{2}+\frac{\sqrt{3}}{2} \beta\right)
$$

Thus, depending on the selected $\beta$ among the four possible values, the model may include from neutral to doubled charged heavy leptons:

$$
q_{E}=[-2,-1,0,+1] \text { for } \beta=\left[\sqrt{3}, \frac{1}{\sqrt{3}},-\frac{1}{\sqrt{3}},-\sqrt{3}\right] \text {. }
$$

From phenomenological reasons we are also interested in heavy neutral particles. The section 2.2 resolve, in the context of scalars, that the most general potential can be formulated regardless the sign of $\beta$, i.e. it is sufficient to consider each of the two possible modulus. Thus, in the remainder of this work we probe those models where $\beta=\left[-\sqrt{3},-\frac{1}{\sqrt{3}}\right]$.

Finally, let us summarize the general hypercharge of triplets and anti-triplets:

- Leptons $\psi_{\alpha}:\left(\mathbf{1}, \mathbf{3},-\left(\frac{1}{2}+\frac{\beta}{2 \sqrt{3}}\right)\right)$

- Quarks $Q_{i}:\left(\mathbf{3}, \overline{\mathbf{3}}, \frac{1}{6}+\frac{\beta}{2 \sqrt{3}}\right)$

- Quarks $Q_{3}$ : $\left(\mathbf{3}, \mathbf{3}, \frac{1}{6}-\frac{\beta}{2 \sqrt{3}}\right)$

- $u_{a}^{R}:\left(\mathbf{3}, \mathbf{1}, \frac{2}{3}\right)$

- $d_{a}^{R}:\left(3,1,-\frac{1}{3}\right)$

- $J_{i}^{R}:\left(3,1, \frac{1}{6}+\beta \frac{\sqrt{3}}{2}\right)$

- $\mathrm{J}_{3}^{R}:\left(3,1, \frac{1}{6}-\beta \frac{\sqrt{3}}{2}\right)$

- $l_{\alpha}^{R}:(\mathbf{1}, \mathbf{1},-1)$

- $E_{\alpha}^{R}:\left(\mathbf{1}, \mathbf{1},-\left(\frac{1}{2}+\beta \frac{\sqrt{3}}{2}\right)\right)$, 
where $\alpha=[e, \mu, \tau], a=[1,2,3]$ and $i=[1,2]$. In the $3-3-1 \mathrm{HL}$ it is required the same number of fermions triplets in the fundamental and conjugated representation in order to achieve anomaly cancellations. The condition permits a freedom of choice to put the first two quark generations in the fundamental representation, while leptons and $Q_{3}$ are left in the conjugated one. In this framework the SM quarks and leptons must be switched inside the triplets and the above $\mathrm{X}$-hypercharges amended by $\beta \rightarrow-\beta$. Once both signs for $\beta$ are considered in the present work, this additional variant must render an equivalent phenomenology and has already been considered in [1].

We conclude this section with a brief comment on the Scalar triplets. The gauge-fixing Lagrangian, discussed in section 2.4, in general is defined from the kinetic sector of the Scalars and contain terms proportional to their product with a correspondent gauge boson. For example, the first line of the product $D^{\mu} \chi$ contains the sum $\partial^{\mu} \chi_{V}+u V$ such that its squared will produce the bilinear $u V \partial \chi_{V}$. Therefore, we introduce the following notation intending to leave explicit how the gauge-structure of the model will connect the scalars with vector bosons:

$$
\begin{aligned}
& \chi=\left(\begin{array}{c}
\chi^{V} \\
\chi^{U} \\
\frac{u+\bar{H}+\mathrm{i} \chi_{g}}{\sqrt{2}}
\end{array}\right), \quad\left(\mathbf{1}, \mathbf{3}, \frac{\beta}{\sqrt{3}}\right) \\
& \rho=\left(\begin{array}{c}
\rho^{W} \\
\frac{v_{\rho}+H_{\rho}+\mathrm{i} \rho_{g}}{\sqrt{2}} \\
\rho^{-U}
\end{array}\right), \quad\left(\mathbf{1}, \mathbf{3}, \frac{1}{2}-\frac{\beta}{2 \sqrt{3}}\right) \\
& \eta=\left(\begin{array}{c}
v_{\eta}+H_{\eta}+\mathrm{i} \eta_{g} \\
\sqrt{2} \\
\eta^{-W} \\
\eta^{-V}
\end{array}\right), \quad\left(\mathbf{1}, \mathbf{3},-\left(\frac{1}{2}+\frac{\beta}{2 \sqrt{3}}\right)\right),
\end{aligned}
$$

where the minus sign indicates that their charges are in opposite sign as those defined in equation (2.33). As will be repeatedly explored, when these connections are not broken by $\beta$ dependent terms, they may control the pattern of scalar mixing and correspond to residual symmetries that avoid the heavy leptons to decay. These features, along with their phenomenological consequences, have been addressed by the authors in [1], and here we complement it by exposing the vertices allowed by the gauge symmetry that could create decaying channels into the light asymptotic fields. Therefore, the complete set of elements for the potential are considered and a $Z_{2}$ symmetry is not assumed a priori. We demonstrate that the pattern of scalar mixing present in the incomplete and universal ${ }^{4}$ potential eliminates the totality of tree-level interactions, leaving the heavy-lepton stable.

\subsection{Self-interactions of scalars}

Here we remark and examine the algebraic path followed by the symmetry breaking of a generic potential which may conduce the gauge theories to their correct spectra. We can start then by presenting some aspects of this sector in the Standard Model, where:

4 The term 'universal' refers to the components present in all versions of the model. 


$$
V(\Phi)=\mu \Phi^{\dagger} \Phi+\lambda\left(\Phi^{\dagger} \Phi\right)^{2}
$$

and

$$
\Phi=\left(\varphi_{g}^{+} \frac{\varphi^{0}+\mathrm{i} \varphi_{g}^{0}}{\sqrt{2}}\right)^{\top}
$$

The subindex ' $\mathrm{g}$ ' on some of the above fields is referring to the Goldstone bosons. In fact, an intermediate step along the development of the Goldstone theorem is supported by the condition that the vacuum of the theory is located on the point where one of the neutral fields, namely $\varphi^{0}$, assume a value different from zero. This vacuum stability condition (VSC) will correspond to one or a set of equations that, when applied back to equation (2.41), cancel the mass terms for these ' $g$ ' degrees of freedom. The contribution to the mass of this particles will be gauge-dependent and comes from the insertion of a gauge-fixing Lagrangian. That is what we called the path, effectively traced by the theorem, for identifying these massless particles.

The vev is usually denoted by $v$, such that the vacuum condition can be read like

$$
\left.\frac{\partial V(\Phi)}{\partial \varphi^{0}}\right|_{\left\langle\varphi^{0}\right\rangle=v}=0
$$

The minimum point represented as $\left\langle\varphi^{0}\right\rangle=v$ denotes that the derivative is being taken on the fields vev. The $\varphi^{0}$ is then expanded around its vev, $\varphi^{0}=v+H$, and the above condition rewritten as:

$$
\left.\frac{\partial H}{\partial \phi^{0}} \frac{\partial V}{\partial H}\right|_{\langle H\rangle=0}=0, \quad \text { or, shortly, }\left.\quad \partial_{H} V\right|_{H=0}=0 .
$$

The potential $V(H)$ is a polynomial and the equation (2.44) implies, therefore, that its linear term on $H$ must vanish, or

$$
\mu+\lambda v^{2}=0
$$

which enables us to rewrite equation $(2.41)$ as

$$
V(\Phi)=\lambda\left(\Phi^{\dagger} \Phi-\frac{v^{2}}{2}\right)^{2}
$$

Thus, the zeroth order term vanishes inside the brackets, leaving only the Higgs with a mass term in the function.

The VSC defines a set of equations which will give us the correct mass matrix coming from the potential. This is seen, for example, in the simplest one dimensional case of the SM. The rotation to the mass eigenstates must also include the contribution coming from quadratic mixed terms present in the gauge-kinetic scalar Lagrangian. These terms are strictly correlated to the gauge-fixing piece, whose treatment leads to the correct mass of the Goldstone bosons. Finally, the Goldstone theorem is essentially based on the vacuum stability and reveal, as a corollary, that these mass matrices, i.e. those coming from the potential and the gauge-fixing, must reside in orthogonal subspaces. On what follows, this result is treated in detail.

By focusing on the potential, it might be important to consider the whole spectra of scalar fields. In order to the standard quarks and leptons acquire their masses, two additional triplets are introduced with the following components: 


$$
\beta=-\sqrt{3}: \rho=\left(\begin{array}{c}
\Phi_{\rho} \\
\rho_{U}^{++}
\end{array}\right)(\mathbf{1}, \mathbf{3}, 1), \quad \eta=\left(\begin{array}{c}
\tilde{\Phi}_{\eta} \\
\eta_{V}^{+}
\end{array}\right) \quad(\mathbf{1}, \mathbf{3}, 0),
$$

where $\Phi_{\rho}$ and $\tilde{\Phi}_{\eta}$ are just denoting that these fields have a similar structure to the doublet in equation (2.42) and its conjugate. The expansion of $\chi^{0}$ around its vev, $\chi^{0}=u+\bar{H}$, introduces the new heavy Higgs, $\bar{H}$. As a matter of counting, we are going to identify those eight scalar fields which are connected, as additional degrees of freedom, to the vectors $V^{ \pm}, U^{ \pm \pm}, W^{ \pm}, Z^{\prime}$ and $Z$.

It has been emphasized in (2.4) that the choice of a specific gauge can be made independently for each gauge interaction. Through their couplings with the SM gauge bosons, the fields in $\Phi_{\rho}$ and $\tilde{\Phi}_{\eta}$ settle a pattern of mixing that will be followed by the composition of the potential presented below. Thus, it may be more elucidative to keep developing the model under an arbitrary gauge. We define, for $\beta=-\sqrt{3}$,

$$
\rho=\left(\begin{array}{c}
\rho_{W}^{+} \\
\left(v_{\rho}+H_{\rho}\right)+i \rho_{g} \\
\hline \sqrt{2} \\
\rho_{U}^{++}
\end{array}\right), \quad \eta=\left(\begin{array}{c}
\frac{\left(v_{\eta}+H_{\eta}\right)+i \eta_{g}}{\sqrt{2}} \\
\eta_{W}^{-} \\
\eta_{V}^{+}
\end{array}\right)
$$

and finally introduce the most general $\beta$-independent potential [5]

$$
\begin{aligned}
V(\chi, \rho, \eta)= & \mu_{\chi} \chi^{\dagger} \chi+\lambda_{\chi}\left(\chi^{\dagger} \chi\right)^{2}+\mu_{\eta} \eta^{\dagger} \eta+\lambda_{\eta}\left(\eta^{\dagger} \eta\right)^{2}+\mu_{\rho} \rho^{\dagger} \rho+\lambda_{\rho}\left(\rho^{\dagger} \rho\right)^{2} \\
& +\lambda_{\rho \eta}\left(\rho^{\dagger} \rho\right)\left(\eta^{\dagger} \eta\right)+\lambda_{\rho \chi}\left(\rho^{\dagger} \rho\right)\left(\chi^{\dagger} \chi\right)+\lambda_{\eta \chi}\left(\eta^{\dagger} \eta\right)\left(\chi^{\dagger} \chi\right) \\
& +\bar{\lambda}_{\rho \eta}\left(\rho^{\dagger} \eta\right)\left(\eta^{\dagger} \rho\right)+\bar{\lambda}_{\rho \chi}\left(\rho^{\dagger} \chi\right)\left(\chi^{\dagger} \rho\right)+\bar{\lambda}_{\eta \chi}\left(\eta^{\dagger} \chi\right)\left(\chi^{\dagger} \eta\right) \\
& +\sqrt{2} \zeta\left(\epsilon_{i j k} \rho^{i} \eta^{j} \chi^{k}+\text { h.c. }\right) .
\end{aligned}
$$

The first two entries of each triplet define a $S U(2)$ doublet, such that any of the above terms are $S U(3), S U(2), U(1)_{X}, U(1)_{Y}$ invariant. In addition, from the definitions of equation (2.40), there can also exist a set of new terms added to equation (2.49), in the context of specific values for $\beta$. In other words, depending on the specific model being regarded, new elements might emerge to the potential.

\subsection{Vacuum stability condition}

In this framework, the vacuum stability condition is given by

$$
\begin{aligned}
& \left.\frac{\partial V\left(\chi^{0}, \rho^{0}, \eta^{0}\right)}{\partial \chi^{0}}\right|_{\left\langle\chi^{0}\right\rangle=u}=0,\left.\quad \frac{\partial V\left(\chi^{0}, \rho^{0}, \eta^{0}\right)}{\partial \rho^{0}}\right|_{\left\langle\rho^{0}\right\rangle=v_{\rho}}=0,\left.\quad \frac{\partial V\left(\chi^{0}, \rho^{0}, \eta^{0}\right)}{\partial \eta^{0}}\right|_{\left\langle\eta^{0}\right\rangle=v_{\eta}} \\
& \quad=0,
\end{aligned}
$$

where $\rho^{0}$ and $\eta^{0}$ denote the neutral component of the respective triplet. The relations above are translated into

$$
\begin{aligned}
& \left.\partial_{\bar{H}} V\left(\bar{H}, H_{\rho}, H_{\eta}\right)\right|_{\bar{H}=0}=0,\left.\quad \partial_{H_{\rho}} V\left(\bar{H}, H_{\rho}, H_{\eta}\right)\right|_{H_{\rho}=0}=0, \\
& \left.\partial_{H_{\eta}} V\left(\bar{H}, H_{\rho}, H_{\eta}\right)\right|_{H_{\eta}=0}=0
\end{aligned}
$$


i.e. the coefficient of linear terms must vanish. For illustration, a geometrical picture can be drawn to the potential vacuum as follows. First, let us rewrite equation (2.49) as

$$
\begin{aligned}
V(\chi, \rho, \eta)= & \left.V\right|_{\rho, \eta=0}+\left.V\right|_{\chi, \eta=0}+\left.V\right|_{\chi, \rho=0} \\
& +\lambda_{\rho \eta}\left(\rho^{\dagger} \rho\right)\left(\eta^{\dagger} \eta\right)+\lambda_{\rho \chi}\left(\rho^{\dagger} \rho\right)\left(\chi^{\dagger} \chi\right)+\lambda_{\eta \chi}\left(\eta^{\dagger} \eta\right)\left(\chi^{\dagger} \chi\right) \\
& +\bar{\lambda}_{\rho \eta}\left(\rho^{\dagger} \eta\right)\left(\eta^{\dagger} \rho\right)+\bar{\lambda}_{\rho \chi}\left(\rho^{\dagger} \chi\right)\left(\chi^{\dagger} \rho\right)+\bar{\lambda}_{\eta \chi}\left(\eta^{\dagger} \chi\right)\left(\chi^{\dagger} \eta\right) \\
& +\sqrt{2} \zeta\left(\epsilon_{i j k} \rho^{i} \eta^{j} \chi^{k}+\text { h.c. }\right) .
\end{aligned}
$$

The first line of equation (2.52) is composed by the three functions defining the scenario where the symmetry breaking occur independently, i.e. when just one of the triplets are present in the theory. The $\left.V\right|_{\rho, \eta=0}$, for instance, comprises the piece regarding solely the breaking $S U(3)_{L} \otimes U(1)_{X} \rightarrow S U(2)_{L} \otimes U(1)_{Y}$. The vacuum condition to it provides

$$
\left.\partial_{\bar{H}}\left(\left.V\right|_{\rho, \eta=0}\right)\right|_{\bar{H}=0}=0 \quad \rightarrow \quad \mu_{\chi}+\lambda_{\chi} u^{2}=0 .
$$

Now, when the VSC is also assumed for the second and third terms of the first line, it implies

$$
\begin{array}{ll}
\left.\partial_{H_{\rho}}\left(\left.V\right|_{\chi, \eta=0}\right)\right|_{H_{\rho}=0}=0 & \rightarrow \quad \mu_{\rho}+\lambda_{\rho} v_{\rho}^{2}=0 \\
\left.\partial_{H_{\eta}}\left(\left.V\right|_{\chi, \rho=0}\right)\right|_{H_{\eta}=0}=0 & \rightarrow \quad \mu_{\eta}+\lambda_{\eta} v_{\eta}^{2}=0 .
\end{array}
$$

When both equations (2.53) and (2.54) are taken, we are in fact assuming that the points $\left(\chi^{0}, \rho^{0}, \varphi^{0}\right)=\left[(u, 0,0),\left(0, v_{\rho}, 0\right),\left(0,0, v_{\eta}\right)\right]$ are either local or global minimum of $V(\chi, \rho$, $\eta)$, along with the point $\left(u, v_{\rho}, v_{\eta}\right)$. Thus, the vacuum could be described by the following ellipsoid

$$
x^{2}+y^{2}+z^{2}+x y+x z+y z-\frac{\left(e^{2}+u^{2}\right)}{u} x-\frac{\left(e^{2}+v_{\rho}^{2}\right)}{v_{\rho}} y-\frac{\left(e^{2}+v_{\eta}^{2}\right)}{v_{\eta}} z+e^{2}=0,
$$

where $e^{2}=\frac{u v_{\rho}+v_{\rho} v_{\eta}+u v_{\eta}}{2}$ and $(x, y, z) \in\left[(u, 0,0),\left(0, v_{\rho}, 0\right),\left(0,0, v_{\eta}\right),\left(u, v_{\rho}, v_{\eta}\right)\right]$ defines a subset of possible solutions.

Finally, by applying equation (2.51) simultaneously to the total potential we are left with the ultimate equations

$$
\begin{aligned}
& \left.\partial_{\bar{H}} V\right|_{\bar{H}=0}=0 \quad \rightarrow \quad \mu_{\chi}+\lambda_{\chi} u^{2}+\frac{v_{\eta}^{2}}{2} \lambda_{\eta \chi}+\frac{v_{\rho}^{2}}{2} \lambda_{\rho \chi}-\frac{v_{\rho} v_{\eta}}{u} \zeta=0 \\
& \left.\partial_{H_{\rho}} V\right|_{H_{\rho}=0}=0 \quad \rightarrow \quad \mu_{\rho}+\lambda_{\rho} v_{\rho}^{2}+\frac{u^{2}}{2} \lambda_{\rho \chi}+\frac{v_{\eta}^{2}}{2} \lambda_{\rho \eta}-\frac{u v_{\eta}}{v_{\rho}} \zeta=0
\end{aligned}
$$

and

$\left.\partial_{H_{\eta}} V\right|_{H_{\eta}=0}=0 \quad \rightarrow \quad \mu_{\eta}+\lambda_{\eta} v_{\eta}^{2}+\frac{u^{2}}{2} \lambda_{\eta \chi}+\frac{v_{\rho}^{2}}{2} \lambda_{\rho \eta}-\frac{u v_{\rho}}{v_{\eta}} \zeta=0$. 
As will be detailed, these three conditions are sufficient to leave the model with a well defined mass spectrum to the scalars.

\subsection{Gauge-fixing Lagrangian}

The gauge interactions of the scalars come from the invariant kinetic term

$$
\mathcal{L}_{s} \supset\left(D_{\mu} \chi\right)^{\dagger}\left(D^{\mu} \chi\right)
$$

For $\beta=-\sqrt{3}$, the triplet can be defined as

$$
\chi=\left(\left(\chi_{g}^{-} \chi_{g}^{--}\right) \frac{\chi_{u}^{0}+i \chi_{g}}{\sqrt{2}}\right)^{\top}
$$

and the $g$ index emphasizes that those states are exactly the Goldstone bosons in the context of a single breaking scale. The Gell-Mann-Nishijima relation of equation (2.4a) also promptly implies $X_{\chi}=-1$.

The covariant derivative (2.31), in matrix form, is given by

$$
\begin{aligned}
D_{\mu}= & \mathbb{I} \partial_{\mu}+\mathrm{i} \frac{g}{\sqrt{2}}\left(\begin{array}{ccc}
0 & W^{+} & V^{-} \\
W^{-} & 0 & U^{--} \\
V^{+} & U^{++} & 0
\end{array}\right) \\
& +\mathrm{i} \operatorname{diag}\left[\left(\begin{array}{ccc}
-e & \frac{g\left(\frac{1}{2}+s_{w}^{2}\right)}{c_{w}} & g\left(\frac{c_{x}}{2 \sqrt{3}}-\sqrt{3} \operatorname{tg}_{w} \operatorname{tg}_{x}\right) \\
-2 e \frac{g\left(-\frac{1}{2}+2 s_{w}^{2}\right)}{c_{w}} & g\left(\frac{c_{x}}{2 \sqrt{3}}-\sqrt{3} \operatorname{tg}_{w} \operatorname{tg}_{x}\right) \\
0 & 0 & g\left(-\frac{c_{x}}{\sqrt{3}}-\sqrt{3} \operatorname{tg}_{w} \operatorname{tg}_{x}\right)
\end{array}\right]\left(\begin{array}{c}
A \\
Z \\
Z^{\prime}
\end{array}\right)\right]
\end{aligned}
$$

where 'diag' requires the vector inside the brackets to define a diagonal $3 \times 3$ matrix. The equation (2.31) provides a direct representation where we can see how the gauge interactions play with the isospin components of the triplets, aside from the manifest separation into the SM and new physics terms, one of the main purposes of this work. Nevertheless, in this section we search for the complete vertices concerning scalars and vectors, and the expansion in equation (2.59) can make their identification simpler.

From the complete result of equation (2.57) only the terms that will indeed be related in our analysis must be selected. First, they include those canceled out by the gauge-fixing Lagrangian. Since each factor in $D_{\mu} \chi$ is at least linear in the fields, we set up the quadratic parts plus all the $\chi_{u}^{0}$ dependent ones, which yield mass to the gauge bosons and introduce the new physical Higgs $\bar{H}$. This corresponds to

$$
\begin{aligned}
\left(D_{\mu} \chi\right)^{\dagger}\left(D^{\mu} \chi\right)= & \frac{1}{2}\left(\partial \chi_{u}^{0}-\mathrm{i} \partial \chi_{g}^{0}-\mathrm{in}_{33} \chi_{u}^{0} Z^{\prime}\right)\left(\partial \chi_{u}^{0}+\mathrm{i} \partial \chi_{g}^{0}+\mathrm{in}_{33} \chi_{u}^{0} Z^{\prime}\right) \\
& +\left(\partial \chi_{g}^{-}+\mathrm{i} \frac{g}{2} \chi_{u}^{0} V^{-}\right)\left(\partial \chi_{g}^{+}-\mathrm{i} \frac{g}{2} \chi_{u}^{0} V^{+}\right) \\
& +\left(\partial \chi_{g}^{--}+\mathrm{i} \frac{g}{2} \chi_{u}^{0} U^{--}\right)\left(\partial \chi_{g}^{++}-\mathrm{i} \frac{g}{2} \chi_{u}^{0} U^{++}\right) \\
& +\mathcal{O}(\text { non }- \text { quadratic terms }) \quad(\beta=-\sqrt{3})
\end{aligned}
$$


where $n_{33} \equiv g\left(-\frac{c_{x}}{\sqrt{3}}-\sqrt{3} \operatorname{tg}_{w} \operatorname{tg}_{x}\right)$. Our final vertices will be given by:

$$
\begin{aligned}
\left(D_{\mu} \chi\right)^{\dagger}\left(D^{\mu} \chi\right)= & \left(\partial \chi_{u}^{0}\right)\left(\partial \chi_{u}^{0}\right)+\left(\partial \chi_{g}^{0}\right)\left(\partial \chi_{g}^{0}\right) \\
& +\left(\partial \chi_{g}^{-}\right)\left(\partial \chi_{g}^{+}\right)+\left(\partial \chi_{g}^{--}\right)\left(\partial \chi_{g}^{++}\right) \\
& +\frac{g^{2}}{4} \chi_{u}^{0} \chi_{u}^{0} V^{-} V^{+}+\frac{g^{2}}{4} \chi_{u}^{0} \chi_{u}^{0} U^{--} U^{++} \\
& +\frac{n_{33}^{2}}{2} \chi_{u}^{0} \chi_{u}^{0} Z^{\prime} Z^{\prime}+n_{33}\left(\partial \chi_{g}^{0}\right) \chi_{u}^{0} Z^{\prime} \\
& +\mathrm{i} \frac{g}{2}\left(\partial \chi_{g}^{+}\right) \chi_{u}^{0} V^{-}-\mathrm{i} \frac{g}{2}\left(\partial \chi_{g}^{-}\right) \chi_{u}^{0} V^{+} \\
& +\mathrm{i} \frac{g}{2}\left(\partial \chi_{g}^{++}\right) \chi_{u}^{0} U^{--}-\mathrm{i} \frac{g}{2}\left(\partial \chi_{g}^{--}\right) \chi_{u}^{0} U^{++} \\
& +\mathcal{O}(\text { non }- \text { quadratic terms }) \quad(\beta=-\sqrt{3}) .
\end{aligned}
$$

We remember that the gauge-fixing factor $\xi_{\mathrm{GB}}$ may be defined independently for each gauge-boson and consider our whole analysis in the arbitrary t'Hooft gauge. The total Lagrangian to the scalars is given by

$$
\mathcal{L}_{s}=\left(D_{\mu} \chi\right)^{\dagger}\left(D^{\mu} \chi\right)+\left(D_{\mu} \eta\right)^{\dagger}\left(D^{\mu} \eta\right)+\left(D_{\mu} \rho\right)^{\dagger}\left(D^{\mu} \rho\right)
$$

and accounts for the gauge-bosons acquisition of mass. At this point we find one of the possible arguments to explain the absence of connection with the SM through non-diagonal interactions. First, let us designate the pairs 12, 13, 23 of the triplet entries in equation (2.40) as the first, second and third doublets, respectively. Now, since the generators $\mathbb{I}_{ \pm}$will act only in the first doublet, the above Lagrangian always present the vertices with the 3-component in pairs of exotic particles, forbidding all the tree-level contributions.

From equation (2.61) one quadratic mixed term is extracted as

$$
\mathcal{L}_{s} \supset u \frac{g}{2}\left(\partial \chi_{V}^{+}\right) V^{-}-u \frac{g}{2}\left(\partial \chi_{V}^{-}\right) V^{+}
$$

These interactions, in general, are related to a transversal propagator for the gauge-bosons and can be equivalently replaced via the insertion of a gauge-fixing Lagrangian. Apart from that, the scalars involved in the second breaking will also contribute to the mass of $V$ and $U$, including a similar momentum-dependent vertex

$$
\mathcal{L}_{s} \supset v_{\eta} \frac{g}{2}\left(\partial \eta_{V}^{-}\right) V^{+}-v_{\eta} \frac{g}{2}\left(\partial \eta_{V}^{+}\right) V^{-} .
$$

The Goldstones will be identified after the rotation of the correlated degrees of freedom inside $\chi$ and $\eta$. To the above case, it can be achieved via the orthogonal matrix

$$
R_{\eta \chi}^{V}=\frac{1}{\bar{u}_{\eta}}\left(\begin{array}{cc}
u & -v_{\eta} \\
v_{\eta} & u
\end{array}\right), \quad \bar{u}_{\eta}=\sqrt{u^{2}+v_{\eta}^{2}}
$$

or

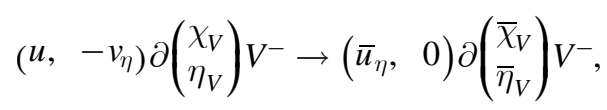


where

$$
\left(\begin{array}{l}
\bar{\chi}_{V} \\
\bar{\eta}_{V}
\end{array}\right)=R_{\eta \chi}^{V}\left(\begin{array}{c}
\chi_{V} \\
\eta_{V}
\end{array}\right)
$$

From equation (2.66) the $\bar{\chi}_{V}$ can be identified as the Goldstone of our theory. The procedure thus described follows from the group structure and is independent of $\beta$. In section 2.7 it will be seen that the rotation equation (2.67) defines a larger block diagonal matrix that plays an important role on the diagonalization of the total mass matrix. After the insertion of additional self-interactions, the potential acquires a new structure of mixing that differs from the gaugefixing mass matrix. However, we may consistently make use of a general consequence of the Goldstone theorem which constrains these two components to lie on orthogonal subspaces, and then verify that our previous knowledge about the rotation of $\mathbb{M}_{\xi}$ can simplify the procedure of finding the total mass matrix, $\mathbb{M}_{s}$, diagonal.

In the context of equation (2.52), the new matrix on the basis $\left(\chi_{V} \eta_{V}\right)$ will appear like

$$
\left(\begin{array}{cc}
\mu_{\chi}+\lambda_{\chi} u^{2}+\frac{v_{\eta}^{2}}{2} \bar{\lambda}_{\eta \chi}+\frac{v_{\eta}^{2}}{2} \lambda_{\eta \chi}+\frac{v_{\rho}^{2}}{2} \lambda_{\rho \chi} & \frac{u v_{\eta}}{2} \bar{\lambda}_{\eta \chi}+v_{\rho} \zeta \\
\frac{u v_{\eta}}{2} \bar{\lambda}_{\eta \chi}+v_{\rho} \zeta & \mu_{\eta}+\lambda_{\eta} v_{\eta}^{2}+\frac{u^{2}}{2} \bar{\lambda}_{\eta \chi}+\frac{u^{2}}{2} \lambda_{\eta \chi}+\frac{v_{\rho}^{2}}{2} \lambda_{\rho \eta}
\end{array}\right)
$$

which can be simplified by applying the VSC of equation (2.56) to

$$
\begin{aligned}
V(\chi, \rho, \eta) & \supset\left(\begin{array}{ll}
\chi_{V}^{*} & \eta_{V}^{*}
\end{array}\right)\left(\begin{array}{ll}
\frac{v_{\eta}^{2}}{2} \bar{\lambda}_{\eta \chi}+\frac{v_{\rho} v_{\eta}}{u} \zeta & \frac{u v_{\eta}}{2} \bar{\lambda}_{\eta \chi}+v_{\rho} \zeta \\
\frac{u v_{\eta}}{2} \bar{\lambda}_{\eta \chi}+v_{\rho} \zeta & \frac{u^{2}}{2} \bar{\lambda}_{\eta \chi}+\frac{u v_{\rho}}{v_{\eta}} \zeta
\end{array}\right)\left(\begin{array}{l}
\chi_{V} \\
\eta_{V}
\end{array}\right) \\
= & \frac{\lambda_{V}}{2}\left(\begin{array}{ll}
\chi_{V}^{*} & \eta_{V}^{*}
\end{array}\right)\left(\begin{array}{cc}
v_{\eta}^{2} & u v_{\eta} \\
u v_{\eta} & u^{2}
\end{array}\right)\left(\begin{array}{l}
\chi_{V} \\
\eta_{V}
\end{array}\right)
\end{aligned}
$$

where

$$
\frac{\lambda_{V}}{2}=\frac{\bar{\lambda}_{\eta \chi}}{2}+\frac{v_{\rho} \zeta}{u v_{\eta}}
$$

The matrix in equation (2.69) can also be directly diagonalized via $R_{\eta \chi}^{V}$, leading to

$$
\frac{\lambda_{V}}{2}\left(\begin{array}{cc}
\bar{\chi}_{V}^{*} & \bar{\eta}_{V}^{*}
\end{array}\right)\left(\begin{array}{cc}
0 & 0 \\
0 & \bar{u}_{\eta}^{2}
\end{array}\right)\left(\begin{array}{l}
\bar{\chi}_{V} \\
\bar{\eta}_{V}
\end{array}\right) \quad \rightarrow \quad m_{\eta}^{2}=\lambda_{V} \bar{u}_{\eta}^{2} .
$$

The Goldstone theorem is then being manifest. The quadratic mass coming from the gauge-fixing Lagrangian is

$$
\mathbb{M}_{\xi}^{2}=\xi_{V}\left(\begin{array}{cc}
u^{2} & -u v_{\eta} \\
-u v_{\eta} & v_{\eta}^{2}
\end{array}\right) \rightarrow \mathbb{M}_{\xi}^{2} \cdot\left(\begin{array}{cc}
v_{\eta}^{2} & u v_{\eta} \\
u v_{\eta} & u^{2}
\end{array}\right)=\mathbb{O}
$$

where its orthogonality to the matrix in equation (2.69) has been registered.

\subsection{Gauge-boson masses}

The gauge-dependent mass matrix is sufficient to exhibit the gauge-boson masses which, for completeness, are presented here as extracted from the scalar Lagrangian: 
$m_{W}^{2}=\frac{g^{2}}{4} \bar{v}^{2}, \quad m_{V}^{2}=\frac{g^{2}}{4} \bar{u}_{\rho}^{2}, \quad m_{U}^{2}=\frac{g^{2}}{4} \bar{u}_{\eta}^{2}, \quad m_{Z}^{2}=\frac{g^{2}}{4 c_{w}^{2}} \bar{v}^{2}$

In order to be general, we distinguish the $U$ and $V$ masses above. However, on the assumption $u \gg v_{\rho}, v_{\eta}$, their values would be equal and differ from the $Z^{\prime}$ mass via a $\beta$ dependent factor, such that

$$
m_{Z^{\prime}}^{2}=\frac{g^{2}}{3} \frac{u^{2}}{c_{x}^{2}}
$$

The above set of relations can result in predictions for some of the gauge boson-masses. Since $s_{x}^{2}=\beta^{2} \tan ^{2} \theta_{w}$, the equation (2.74) may already reveal a strong constraint to the $|\beta|=\sqrt{3}$ variants in the scenario where the Weinberg angle evolutes to $s_{w}^{2} \sim \frac{1}{4}$, what would force the charged gauge bosons to live in an excluded low-energy regime [13, 21].

The bilinears for neutral vectors are given by

$$
\mathcal{L}_{s} \supset-g \frac{c_{x}}{\sqrt{3}} \partial \chi_{g} Z^{\prime}-g \frac{v_{\rho}}{2 c_{w}} \partial \rho_{g} Z+g \frac{v_{\eta}}{2 c_{w}} \partial \eta_{g} Z
$$

corresponding, after the $\mathcal{L}_{g . f}$. insertion, to the correct $\xi$-dependent $Z, Z^{\prime}$ propagators and a new mass matrix to the scalars:

$$
\left.\mathbb{M}_{\xi}^{2}\right|_{Z, Z^{\prime}}=\left(\begin{array}{cc}
\frac{\xi_{Z}}{4 c_{w}^{2}}\left(\begin{array}{cc}
v_{\eta}^{2} & -v_{\eta} v_{\rho} \\
-v_{\eta} v_{\rho} & v_{\rho}^{2}
\end{array}\right) & \mathbf{0} \\
\mathbf{0}^{\top} & \xi_{Z^{\prime}} \frac{u^{2}}{3} c_{x}^{2}
\end{array}\right)
$$

on the basis $\left(\eta_{g}, \rho_{g}, \chi_{g}\right)$. It can be verified that the above result is in fact orthogonal to the $\mathbb{P}$ matrix presented in the next section.

\subsection{Scalar masses}

We initiate this section approaching the masses for the remaining scalars. The expansion of equation (2.52) gives us the mixing pattern between the charged particles and a result similar to equation (2.71) must arise. Let us cover this explicitly, but now in a more direct mode.

For the U-type scalars on the basis $\left(\chi_{U} \rho_{U}\right)$ it follows

$$
\left(\begin{array}{cc}
\mu_{\chi}+\lambda_{\chi} u^{2}+\frac{v_{\rho}^{2}}{2} \bar{\lambda}_{\rho \chi}+\frac{v_{\eta}^{2}}{2} \lambda_{\eta \chi}+\frac{v_{\rho}^{2}}{2} \lambda_{\rho \chi} & \frac{u v_{\eta}}{2} \bar{\lambda}_{\rho \chi}+v_{\eta} \zeta \\
\frac{u v_{\eta}}{2} \bar{\lambda}_{\rho \chi}+v_{\eta} \zeta & \mu_{\rho}+\lambda_{\rho} v^{2}+\frac{u^{2}}{2} \bar{\lambda}_{\rho \chi}+\frac{u^{2}}{2} \lambda_{\rho \chi}+\frac{v_{\eta}^{2}}{2} \lambda_{\rho \eta}
\end{array}\right)
$$

such that, with the help of equation (2.56), it can be simplified to

$$
\frac{\lambda_{U}}{2}\left(\begin{array}{ll}
\chi_{U}^{*} & \rho_{U}^{*}
\end{array}\right)\left(\begin{array}{cc}
v_{\rho}^{2} & u v_{\rho} \\
u v_{\rho} & u^{2}
\end{array}\right)\left(\begin{array}{l}
\chi_{U} \\
\rho_{U}
\end{array}\right) \rightarrow \frac{\lambda_{U}}{2}=\frac{\bar{\lambda}_{\rho \chi}}{2}+\frac{v_{\eta} \zeta}{u v_{\rho}}
$$

or

$$
\frac{\lambda_{U}}{2}\left(\begin{array}{cc}
\bar{\chi}_{U}^{*} & \bar{\rho}_{U}^{*}
\end{array}\right)\left(\begin{array}{cc}
0 & 0 \\
0 & \bar{u}_{\rho}^{2}
\end{array}\right)\left(\begin{array}{c}
\bar{\chi}_{U} \\
\bar{\rho}_{U}
\end{array}\right) \quad \rightarrow \quad m_{\rho}^{2}=\lambda_{U} \bar{u}_{\rho}^{2}
$$


where the rotation matrix is given by

$$
R_{\rho \chi}^{U}=\frac{1}{\bar{u}_{\rho}}\left(\begin{array}{cc}
u & -v_{\rho} \\
v_{\rho} & u
\end{array}\right) \quad \rightarrow \quad \bar{u}_{\rho}=\sqrt{u^{2}+v_{\rho}^{2}}
$$

To conclude the non-diagonal sector, there are those scalars coupled with the SM gauge bosons:

$$
\frac{\lambda_{W}}{2}\left(\begin{array}{ll}
\eta_{W}^{*} & \rho_{W}^{*}
\end{array}\right)\left(\begin{array}{cc}
v_{\rho}^{2} & v_{\rho} v_{\eta} \\
v_{\rho} v_{\eta} & v_{\eta}^{2}
\end{array}\right)\left(\begin{array}{l}
\eta_{W} \\
\rho_{W}
\end{array}\right) \rightarrow \frac{\lambda_{W}}{2}=\frac{\bar{\lambda}_{\rho \eta}}{2}+\frac{u \zeta}{v_{\rho} v_{\eta}}
$$

or

$$
\frac{\lambda_{W}}{2}\left(\bar{\eta}_{W}^{*} \bar{\rho}_{W}^{*}\right)\left(\begin{array}{cc}
0 & 0 \\
0 & \bar{v}^{2}
\end{array}\right)\left(\begin{array}{l}
\bar{\eta}_{W} \\
\bar{\rho}_{W}
\end{array}\right) \quad \rightarrow \quad m_{\rho_{W}}^{2}=\lambda_{W} \bar{v}^{2}
$$

Equivalently,

$$
R_{\rho \eta}^{W}=\frac{1}{\bar{v}}\left(\begin{array}{cc}
v_{\eta} & -v_{\rho} \\
v_{\rho} & v_{\eta}
\end{array}\right) \quad \rightarrow \quad \bar{v}=\sqrt{v_{\rho}^{2}+v_{\eta}^{2}}
$$

where

$$
\left(\begin{array}{c}
\bar{\eta}_{W} \\
\bar{\rho}_{W}
\end{array}\right)=R_{\rho \eta}^{W}\left(\begin{array}{c}
\eta_{W} \\
\rho_{W}
\end{array}\right)
$$

From the beginning of our review the $Z^{\prime}$ has been assumed to not mix with $Z$ and photon, a claim justified from the hierarchy between the breaking scales. We may expect, therefore, the same pattern to be also present in the neutral scalar sector. On what follows this feature is identified in detail. First we write the mass matrix of pseudo-scalars on the following basis

$$
\left(\begin{array}{lll}
\eta_{g} & \rho_{g} & \chi_{g}
\end{array}\right) \mathbb{P}\left(\begin{array}{l}
\eta_{g} \\
\rho_{g} \\
\chi_{g}
\end{array}\right)
$$

such that the entries of $\mathbb{P}$ are given by

$$
\left(\begin{array}{ccc}
\frac{1}{2}\left(\mu_{\eta}+\lambda_{\eta} v_{\eta}^{2}+\frac{u^{2}}{2} \lambda_{\eta \chi}+\frac{v_{\rho}^{2}}{2} \lambda_{\rho \eta}\right) & \frac{u \zeta}{2} & \frac{v_{\rho} \zeta}{2} \\
\frac{u \zeta}{2} & \frac{1}{2}\left(\mu_{\rho}+\lambda_{\rho} v_{\rho}^{2}+\frac{u^{2}}{2} \lambda_{\rho \chi}+\frac{v_{\eta}^{2}}{2} \lambda_{\rho \eta}\right) & \frac{v_{\eta} \zeta}{2} \\
\frac{v_{\rho} \zeta}{2} & \frac{v_{\eta} \zeta}{2} & \frac{1}{2}\left(\mu_{\chi}+\lambda_{\chi} u^{2}+\frac{v_{\eta}^{2}}{2} \lambda_{\eta \chi}+\frac{v_{\rho}^{2}}{2} \lambda_{\rho \chi}\right)
\end{array}\right)
$$

or, simply,

$$
\mathbb{P}=\frac{u \zeta}{2}\left(\begin{array}{ccc}
\frac{v_{\rho}}{v_{\eta}} & 1 & \frac{v_{\rho}}{u} \\
1 & \frac{v_{\eta}}{v_{\rho}} & \frac{v_{\eta}}{u} \\
\frac{v_{\rho}}{u} & \frac{v_{\eta}}{u} & \frac{v_{\rho} v_{\eta}}{u^{2}}
\end{array}\right) \approx \frac{u \zeta}{2}\left(\begin{array}{ccc}
\frac{v_{\rho}}{v_{\eta}} & 1 & 0 \\
1 & \frac{v_{\eta}}{v_{\rho}} & 0 \\
0 & 0 & 0
\end{array}\right)=\frac{\lambda_{P}}{2}\left(\begin{array}{ccc}
v_{\rho}^{2} & v_{\rho} v_{\eta} & 0 \\
v_{\rho} v_{\eta} & v_{\eta}^{2} & 0 \\
0 & 0 & 0
\end{array}\right)
$$

We emphasize that the second signal represents the absence of contribution from the lowenergy breaking to the $Z^{\prime}$ mass and must not be understood as a formal limit. The constant is given by 


$$
\lambda_{P}=\frac{u \zeta}{v_{\rho} v_{\eta}} .
$$

Thus, the pseudo-scalars are diagonalized to their mass eigenstates by the same matrix $R_{\rho \eta}^{W}$ of the standard charged scalars. Finally, the potential introduces a single pseudo-scalar, here denoted $\rho_{P}$, such that

$$
\rho_{P}: \quad m_{P}^{2}=\lambda_{P} \bar{v}^{2} .
$$

The presence of this particle is $\beta$-independent.

The real scalars follow a similar analysis. In the basis

$$
\left(\begin{array}{lll}
H_{\eta} & H_{\rho} & \bar{H}
\end{array}\right) \mathbb{S}\left(\begin{array}{l}
H_{\eta} \\
H_{\rho} \\
\bar{H}
\end{array}\right)
$$

and after the equation (2.56) insertion, the $\mathbb{S}$ entries are

$$
\mathbb{S}=\left(\begin{array}{ccc}
\lambda_{\eta} v_{\eta}^{2}+\zeta \frac{u v_{\rho}}{2 v_{\eta}} & \lambda_{\rho \eta} \frac{v_{\eta} v_{\rho}}{2}-\frac{u \zeta}{2} & 0 \\
\lambda_{\rho \eta} \frac{v_{\eta} v_{\rho}}{2}-\frac{u \zeta}{2} & \lambda_{\rho} v_{\rho}^{2}+\zeta \frac{u v_{\eta}}{2 v_{\rho}} & 0 \\
0 & 0 & \lambda_{\chi} u^{2}+\zeta \frac{v_{\eta} v_{\rho}}{2 u}
\end{array}\right) .
$$

The above result includes that, in the context of $u \gg v_{\rho} v_{\eta}$, the third non-diagonal elements are small compared with the remainder, and thus give a neglected contribution to the eigenvalues. Their original values are:

$$
\begin{aligned}
& \mathbb{S}_{13}: \zeta \frac{v_{\rho}}{2}-\frac{v_{\eta}}{u}\left(\mu_{\eta}+\lambda_{\eta} v_{\eta}^{2}\right)-\lambda_{\rho \eta} \frac{v_{\rho}^{2} v_{\eta}}{2 u} \\
& \mathbb{S}_{23}: \zeta \frac{v_{\eta}}{2}-\frac{v_{\rho}}{u}\left(\mu_{\rho}+\lambda_{\rho} v_{\rho}^{2}\right)-\lambda_{\rho \eta} \frac{v_{\eta}^{2} v_{\rho}}{2 u} .
\end{aligned}
$$

The matrix $\mathbb{S}$ can be diagonalized by

$$
R_{S}=\left(\begin{array}{ccc}
c_{s} & -s_{S} & 0 \\
s_{S} & c_{s} & 0 \\
0 & 0 & 1
\end{array}\right)
$$

and their simplified eigenvalues are:

$$
\begin{aligned}
m_{H_{\eta}}^{2} & =\frac{1}{v_{\eta} v_{\rho}}\left(\lambda_{\eta} v_{\eta}^{3} v_{\rho}+\lambda_{\rho} v_{\rho}^{3} v_{\eta}\right) \\
& =\lambda_{\eta} v_{\eta}^{2}+\lambda_{\rho} v_{\rho}^{2} \\
m_{H_{\rho}}^{2} & =\frac{1}{v_{\eta} v_{\rho}}\left(\lambda_{\eta} v_{\eta}^{3} v_{\rho}+\lambda_{\rho} v_{\rho}^{3} v_{\eta}+u \zeta\left(v_{\rho}^{2}+v_{\eta}^{2}\right)\right) \\
& =\lambda_{\eta} v_{\eta}^{2}+\lambda_{\rho} v_{\rho}^{2}+u \zeta \frac{\left(v_{\eta}^{2}+v_{\rho}^{2}\right)}{v_{\eta} v_{\rho}}
\end{aligned}
$$




$$
m_{\bar{H}}^{2}=2 \lambda_{\chi} u^{2}+\zeta \frac{v_{\eta} v_{\rho}}{u} .
$$

Note, therefore, an approximate relation between the scalar and pseudo-scalar masses:

$$
m_{H_{\rho}}^{2}=m_{H_{\eta}}^{2}+m_{P}^{2}
$$

Thus, as in the Standard Model, the VSC prevents the mass terms of some charged scalars arising in the potential, leaving their contribution exclusively in the gauge-fixing Lagrangian. In the previous discussion we identified this feature occurring for the versionindependent 3-3-1HL potential and the VSC leading the model to a correct scalar spectrum. Nevertheless, it has not been yet mentioned about the allowed $\beta$-dependent pieces, for example

$$
\left.V(\chi, \rho, \eta)\right|_{\beta=-\sqrt{3}} \supset V_{\rho \eta}^{\chi} \equiv \lambda_{\rho \eta}^{\chi}\left(\chi^{\dagger} \eta\right)\left(\rho^{\dagger} \eta\right)+\text { h.c. }
$$

which could connect the scalars $\left(\eta_{W}, \rho_{W}\right)$ with $\left(\chi_{V}, \eta_{V}\right)$. In the rest of this work we verify that the omission of a term such as equation (2.99) may imply a complete dissociation of the charged exotic sector with standard particles at tree-level, leaving the Yukawa interactions as the ultimate chance to connect them. In section (2.8) we disclose this possibility and conclude that assuming equation (2.99) is equal to zero it is equivalent to assume a discrete symmetry for this variant of the 3-3-1HL.

\subsection{The potential for particular models}

As mentioned previously, the term $\lambda_{\rho \eta}^{\chi}\left(\chi^{\dagger} \eta\right)\left(\rho^{\dagger} \eta\right)$ could a priori be included in the potential ${ }^{5}$ for $\beta=-\sqrt{3}$, creating a leading order connection with the SM from one additional mixing between the four charged scalars.

First, let us see how the mass matrix will emerge in the basis $\left(\begin{array}{lllll}\eta_{W} & \rho_{W} & \eta_{V} & \chi_{V}\end{array}\right)$ after this insertion:

$$
\mathbb{M}_{V}^{2}=\left(\begin{array}{cc}
\frac{\lambda_{W}}{2}\left(\begin{array}{cc}
v_{\rho}^{2} & v_{\rho} v_{\eta} \\
v_{\rho} v_{\eta} & v_{\eta}^{2}
\end{array}\right) & \lambda_{\rho \eta}^{\chi}\left(\begin{array}{cc}
u v_{\rho} & v_{\eta} v_{\rho} \\
u v_{\eta} & v_{\eta}^{2}
\end{array}\right) \\
\lambda_{\rho \eta}^{\chi}\left(\begin{array}{cc}
u v_{\rho} & u v_{\eta} \\
v_{\eta} v_{\rho} & v_{\eta}^{2}
\end{array}\right) & \frac{\lambda_{V}}{2}\left(\begin{array}{cc}
u^{2} & u v_{\eta} \\
u v_{\eta} & v_{\eta}^{2}
\end{array}\right)
\end{array}\right) .
$$

The determinant of $\mathbb{M}_{V}^{2}$ above is in fact zero, a necessary condition to leave the theory with a well-defined spectrum.

The gauge-fixing Lagrangian also contributes with a gauge-dependent squared mass matrix:

$$
\mathbb{M}_{\xi}^{2}=\left(\begin{array}{cc}
\xi_{W}\left(\begin{array}{cc}
v_{\eta}^{2} & -v_{\rho} v_{\eta} \\
-v_{\rho} v_{\eta} & v_{\rho}^{2}
\end{array}\right) & \mathbb{O} \\
\mathbb{O} & \xi_{V}\left(\begin{array}{cc}
v_{\eta}^{2} & -u v_{\eta} \\
-u v_{\eta} & u^{2}
\end{array}\right)
\end{array}\right) .
$$

As mentioned before, one important consequence of the Goldstone theorem requires that these two matrices must lie in orthogonal subspaces, such that

${ }^{5}$ For $\beta=+\sqrt{3}$ the new term is similar to equation (2.99), with $\rho \leftrightarrow \eta$. 


$$
\mathbb{M}_{V}^{2} \cdot \mathbb{M}_{\xi}^{2}=0 \quad\left(\mathbb{M}_{\xi}^{2} \cdot \mathbb{M}_{V}^{2}=0\right) .
$$

We can check this directly by requesting a simple identity for matrix products. Consider a general even $n \times n$ matrix:

$$
\mathbb{M}=\left(\begin{array}{ll}
\mathbb{M}_{1} & \mathbb{M}_{2} \\
\mathbb{M}_{3} & \mathbb{M}_{4}
\end{array}\right)
$$

where $\mathbb{M}_{k}$ are $\frac{n}{2} \times \frac{n}{2}$. Then, it follows that

$$
\mathbb{A} \cdot \mathbb{B}=\left(\begin{array}{ll}
\left(\mathbb{A}_{1} \cdot \mathbb{B}_{1}+\mathbb{A}_{2} \cdot \mathbb{B}_{3}\right) & \left(\mathbb{A}_{1} \cdot \mathbb{B}_{2}+\mathbb{A}_{2} \cdot \mathbb{B}_{4}\right) \\
\left(\mathbb{A}_{3} \cdot \mathbb{B}_{1}+\mathbb{A}_{4} \cdot \mathbb{B}_{3}\right) & \left(\mathbb{A}_{3} \cdot \mathbb{B}_{2}+\mathbb{A}_{4} \cdot \mathbb{B}_{4}\right)
\end{array}\right)
$$

which can be proved by considering a single element

$$
\mathbb{M}_{i j} \in \begin{cases}\mathbb{M}_{1}, & i \in\left[1, \ldots \frac{n}{2}\right], j \in\left[1, \ldots \frac{n}{2}\right] \\ \mathbb{M}_{2}, & i \in\left[1, \ldots \frac{n}{2}\right], j \in\left[\frac{n}{2}+1, \ldots n\right] \\ \mathbb{M}_{3}, & i \in\left[\frac{n}{2}+1, \ldots n\right], j \in\left[1, \ldots \frac{n}{2}\right] \\ \mathbb{M}_{4}, & i \in\left[\frac{n}{2}+1, \ldots n\right], j \in\left[\frac{n}{2}+1, \ldots n\right]\end{cases}
$$

and the general rule for matrix products, $(\mathbb{A} \cdot \mathbb{B})_{i j}=\mathbb{A}_{i k} \mathbb{B}_{k j}+\mathbb{A}_{i l} \mathbb{B}_{l j}$, with $k \in\left[1, \ldots \frac{n}{2}\right]$ and $l \in\left[\frac{n}{2}+1, \ldots n\right]$. For instance, if $(i j) \in \mathbb{M}_{2}$ then $(\mathbb{A} \cdot \mathbb{B})_{i j}=\left(\mathbb{A}_{1} \cdot \mathbb{B}_{2}+\mathbb{A}_{2} \cdot \mathbb{B}_{4}\right)_{i\left(j-\frac{n}{2}\right)}$. Finally, equation (2.104), for $\mathbb{B}_{2}=\mathbb{B}_{3}=0$, implies

$$
\mathbb{A} \cdot \mathbb{B}=\left(\begin{array}{ll}
\left(\mathbb{A}_{1} \cdot \mathbb{B}_{1}\right) & \left(\mathbb{A}_{2} \cdot \mathbb{B}_{4}\right) \\
\left(\mathbb{A}_{3} \cdot \mathbb{B}_{1}\right) & \left(\mathbb{A}_{4} \cdot \mathbb{B}_{4}\right)
\end{array}\right)
$$

and the demonstration of equation (2.102) follows straightforwardly.

- The result of equation (2.104) can be also extended for odd matrices, and to the $3 \times 3$ case we register one useful identity. First, consider the generic matrix

$$
\mathbb{M}=\left(\begin{array}{ll}
\mathbb{M}_{1} & \mathbf{m}_{2} \\
\mathbf{m}_{3}^{\top} & m_{4}
\end{array}\right)
$$

where $\mathbf{m}$ are two-dimensional vectors and $m_{4}$ is a c-number. Thus,

$$
\mathbb{A} \cdot \mathbb{B}=\left(\begin{array}{cc}
\left(\mathbb{A}_{1} \cdot \mathbb{B}_{1}+\mathbf{a}_{2} \cdot \mathbf{b}_{3}^{\top}\right) & \left(\mathbb{A}_{1} \cdot \mathbf{b}_{2}+\mathbf{a}_{2} b_{4}\right) \\
\left(\mathbf{a}_{3}^{\top} \cdot \mathbb{B}_{1}+a_{4} \mathbf{b}_{3}^{\top}\right) & \mathbf{a}_{3}^{\top} \cdot \mathbf{b}_{2}+a_{4} b_{4}
\end{array}\right) .
$$

Now, let us define the total squared mass

$$
\mathbb{M}_{s}^{2} \equiv \mathbb{M}_{V}^{2}+\mathbb{M}_{\xi}^{2}
$$

From equation (2.102) it follows that

$$
\mathbb{M}_{s}^{2} \cdot \mathbb{M}_{\xi}^{2}=\mathbb{M}_{\xi}^{2} \cdot \mathbb{M}_{s}^{2}=\left(\mathbb{M}_{\xi}^{2}\right)^{2}
$$

i.e. these matrices must commute:

$$
\left[\mathbb{M}_{s}^{2}, \mathbb{M}_{\xi}^{2}\right]=0
$$


and, therefore, there must be a matrix $\mathbb{U}$ able to diagonalize them simultaneously. Apart from that, we have already seen that the matrix

$$
\mathbb{D}=\left(\begin{array}{ccc}
\frac{1}{\bar{v}}\left(\begin{array}{cc}
v_{\eta} & -v_{\rho} \\
v_{\rho} & v_{\eta}
\end{array}\right) & \mathbb{O} \\
\mathbb{O} & \frac{1}{\bar{u}_{\eta}}\left(\begin{array}{cc}
u & v_{\eta} \\
-v_{\eta} & u
\end{array}\right)
\end{array}\right)
$$

is such that

$$
\mathbb{D M}_{\xi}^{2} \mathbb{D}^{\top}=\mathbb{X}_{\xi}^{2}
$$

with $\mathbb{X}_{\xi}^{2}$ diagonal. Thus, from equation (2.111), it follows that $\mathbb{D}\left[\mathbb{M}_{s}^{2}, \mathbb{M}_{\xi}^{2}\right] \mathbb{D}^{\top}=0$, or

$$
\left[\overline{\mathbb{M}}_{s}^{2}, \mathbb{X}_{\xi}^{2}\right]=0, \quad \text { where } \quad \overline{\mathbb{M}}_{s}^{2} \equiv \mathbb{D M}_{s}^{2} \mathbb{D}^{\top}
$$

i.e. the rotation of the total mass matrix by $\mathbb{D}$ will commute with the diagonal mass matrix of the Goldstone bosons. In terms of components the above result can be written as

$$
\sum_{k}\left(\overline{\mathbb{M}}_{s}^{2}\right)_{i k}\left(\mathbb{X}_{\xi}^{2}\right)_{k j}=\sum_{k}\left(\mathbb{X}_{\xi}^{2}\right)_{i k}\left(\overline{\mathbb{M}}_{s}^{2}\right)_{k j}
$$

or

$$
\sum_{k}\left[\left(\overline{\mathbb{M}}_{s}^{2}\right)_{i k} \delta_{k j}\left(\mathbb{X}_{\xi}^{2}\right)_{j j}-\delta_{i k}\left(\overline{\mathbb{M}}_{s}^{2}\right)_{k j}\left(\mathbb{X}_{\xi}^{2}\right)_{i i}\right]=0
$$

and, finally,

$$
\left(\overline{\mathbb{M}}_{s}^{2}\right)_{i j}\left\{\left(\mathbb{X}_{\xi}^{2}\right)_{i i}-\left(\mathbb{X}_{\xi}^{2}\right)_{j j}\right\}=0
$$

Thus, the $\overline{\mathbb{M}}_{s}^{2}$ must emerge into a block diagonal form, whose dimension will agree with the number of eigenvalues inside $\mathbb{X}_{\xi}^{2}$ with multiplicity one.

Our second step consists of finding a matrix $\overline{\mathbb{D}}$ to diagonalize $\overline{\mathbb{M}}_{s}^{2}$ and, in general, is simple to define. Again, from equation (2.114), the matrix $\mathbb{X}_{\xi}^{2}$ would remain block-diagonal after a rotation by $\overline{\mathbb{D}}$, such that

$$
(\overline{\mathbb{D}} \mathbb{D}) \mathbb{M}_{\xi}^{2}(\overline{\mathbb{D}} \mathbb{D})^{\top}=\mathbb{X}_{\xi}^{2} \quad \text { and } \quad(\overline{\mathbb{D}} \mathbb{D}) \mathbb{M}_{s}^{2}(\overline{\mathbb{D}} \mathbb{D})^{\top}=\mathbb{X}_{s}^{2}
$$

with $\mathbb{X}_{s}^{2}$ diagonal.

In other words, the matrix that can simultaneously diagonalize the total and the gaugefixing mass matrices is being constructed by parts, after claiming a general Goldstone theorem corollary. Surely, we just need to look at the total $\mathbb{M}_{s}^{2}$. However, the direct identification of the Goldstone bosons becomes clear and definitive through these steps since we require the gauge-dependent sector to play a role in the process.

Let us apply these results in our particular case, from equations (2.100) and (2.101). The $\mathbb{X}_{\xi}^{2}$ was previously obtained and is given by 


$$
\mathbb{X}_{\xi}^{2}=\left(\begin{array}{ccc}
\xi_{W}\left(\begin{array}{cc}
\bar{v}^{2} & \\
& 0
\end{array}\right) & \mathbb{O} \\
& & \\
\mathbb{O} & \xi_{V}\left(\begin{array}{ll}
0 & \\
& \bar{u}_{\eta}^{2}
\end{array}\right)
\end{array}\right)
$$

and, from equation (2.117), we must expect $(\mathbb{D}) \mathbb{M}_{s}^{2}(\mathbb{D})^{\top}$ to contain a $2 \times 2$ block. In fact,

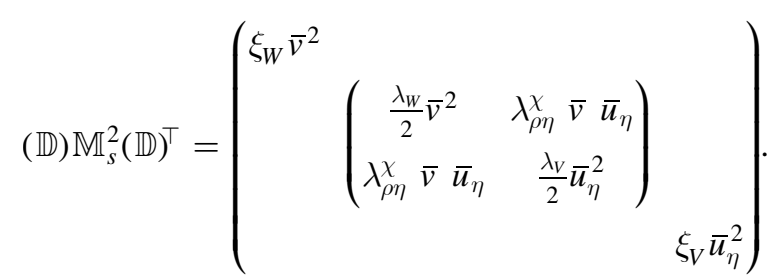

The central matrix can be diagonalized after the insertion of a new $\overline{\mathbb{D}}$, such that

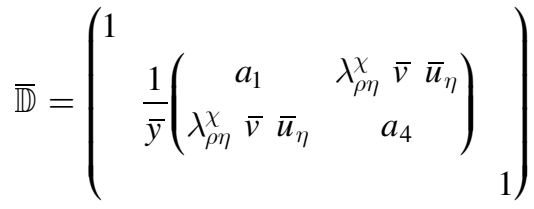

$$
\begin{aligned}
& a_{1} \equiv \frac{1}{2}\left(\frac{\lambda_{W}}{2} \bar{v}^{2}-\frac{\lambda_{V}}{2} \bar{u}_{\eta}^{2}-\sqrt{\left(\frac{\lambda_{W}}{2} \bar{v}^{2}-\frac{\lambda_{V}}{2} \bar{u}_{\eta}^{2}\right)^{2}+\left(2 \lambda_{\rho \eta}^{\chi} \bar{v} \bar{u}_{\eta}\right)^{2}}\right) \\
& a_{4} \equiv \frac{1}{2}\left(\frac{\lambda_{V}}{2} \bar{u}_{\eta}^{2}-\frac{\lambda_{W}}{2} \bar{v}^{2}+\sqrt{\left(\frac{\lambda_{W}}{2} \bar{v}^{2}-\frac{\lambda_{V}}{2} \bar{u}_{\eta}^{2}\right)^{2}+\left(2 \lambda_{\rho \eta}^{\chi} \bar{v} \bar{u}_{\eta}\right)^{2}}\right) \\
& \bar{y} \equiv \sqrt{\left(\lambda_{\rho \eta}^{\chi} \bar{v} \bar{u}_{\eta}\right)^{2}+a_{1}^{2}}
\end{aligned}
$$

and, finally,

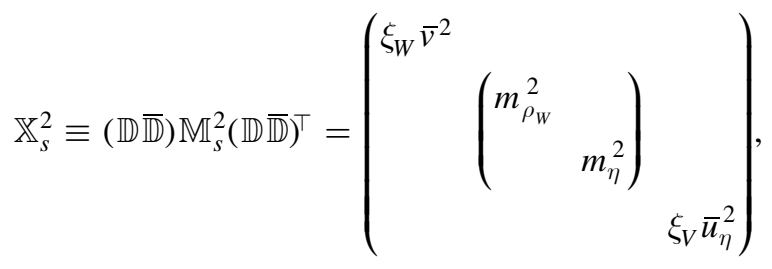

where

$$
\begin{gathered}
m_{\rho_{W}}^{2} \equiv \frac{1}{2}\left(\frac{\lambda_{W}}{2} \bar{v}^{2}+\frac{\lambda_{V}}{2} \bar{u}_{\eta}^{2}-\sqrt{\left(\frac{\lambda_{W}}{2} \bar{v}^{2}-\frac{\lambda_{V}}{2} \bar{u}_{\eta}^{2}\right)^{2}+\left(2 \lambda_{\rho \eta}^{\chi} \bar{v} \bar{u}_{\eta}\right)^{2}}\right) \\
m_{\eta}^{2} \equiv \frac{1}{2}\left(\frac{\lambda_{W}}{2} \bar{v}^{2}+\frac{\lambda_{V}}{2} \bar{u}_{\eta}^{2}+\sqrt{\left(\frac{\lambda_{W}}{2} \bar{v}^{2}-\frac{\lambda_{V}}{2} \bar{u}_{\eta}^{2}\right)^{2}+\left(2 \lambda_{\rho \eta}^{\chi} \bar{v} \bar{u}_{\eta}\right)^{2}}\right)
\end{gathered}
$$

In summary, the $\beta$-independent potential follows a mixing pattern in precise accordance with the $S U(2)$ subalgebras, i.e. by pairs of the scalars entailed by the same gauge-interactions. In the notation of equation (2.40) it corresponds to $\left(\chi^{V} \eta^{V}\right),\left(\chi^{U} \eta^{U}\right)$ and $\left(\rho^{W} \eta^{W}\right)$. When this pattern is broken by the insertion of specific allowed terms, the physical fields 
inside these pairs can be connected through an additional rotation, leaving the gauge-sector unchanged.

The previous analysis of the additional term in the potential holds for $|\beta|=\sqrt{3}$. In addition, from equation $(2.40)$, for $|\beta|=\frac{1}{\sqrt{3}}$ one of the triplets $\rho$ or $\eta$ has the same hypercharge as $\chi$. The authors in [22] introduce a new non- $Z_{2}$ piece in the context of neutral heavy leptons, i.e for $\beta=-\frac{1}{\sqrt{3}}$, where $\chi$ and $\eta$ share the same $X$. These new contributions are given by

$$
\begin{aligned}
& \left.V(\chi, \rho, \eta)\right|_{\beta=-\frac{1}{\sqrt{3}}} \supset V_{\chi \eta} \equiv \mu_{\chi \eta} \chi^{\dagger} \eta+\lambda_{\chi \eta}^{\eta}\left(\chi^{\dagger} \eta\right)\left(\eta^{\dagger} \eta\right)+\lambda_{\chi \eta}^{\rho}\left(\chi^{\dagger} \eta\right)\left(\rho^{\dagger} \rho\right) \\
& +\lambda_{\chi \eta}^{\chi}\left(\chi^{\dagger} \eta\right)\left(\chi^{\dagger} \chi\right)+\text { h.c. }
\end{aligned}
$$

In this variant, the gauge-boson $\mathrm{V}$ has electric charge equals to zero. The above expression presents a linear term in the complex neutral field $\chi_{V}^{0}$, whose coefficient must be zero in order to leave $V(\chi, \rho, \eta)$ with a lower limit at $\left\langle\chi_{V}^{0}\right\rangle=0$. This condition converts into the equation:

$$
\mu_{\chi \eta}+\lambda_{\chi \eta}^{\eta} \frac{v_{\eta}^{2}}{2}+\lambda_{\chi \eta}^{\rho} \frac{v_{\rho}^{2}}{2}+\lambda_{\chi \eta}^{\chi} \frac{u^{2}}{2}=0
$$

Nevertheless, the bilinear $\left(\chi^{\dagger} \eta\right)$ in equation (2.126) turns factorized by the rhs of the above expression, implying that the $\mu_{\chi} \eta$ portion along with the mixing terms for the charged scalars $\left(\chi_{U} \eta_{W}\right)$ will vanish. We rewrite equation (2.126) as

$V_{\chi \eta}=\left.\lambda_{\chi \eta}^{\eta}\left(\chi^{\dagger} \eta\right)\left(\eta^{\dagger} \eta\right)\right|_{v_{\eta}^{2}=0}+\left.\lambda_{\chi \eta}^{\rho}\left(\chi^{\dagger} \eta\right)\left(\rho^{\dagger} \rho\right)\right|_{v_{\rho}^{2}=0}+\left.\lambda_{\chi \eta}^{\chi}\left(\chi^{\dagger} \eta\right)\left(\chi^{\dagger} \chi\right)\right|_{u^{2}=0}+$ h.c.

and emphasize that the notation excludes only the quadratic vev's, preserving the trilinear interactions.

Thus far the exotic sector is still connected to the SM particles through tree-level interactions with real Higgs bosons. There will still be remaining bilinears involving the real scalars and the neutral $\left(\chi_{V}, \eta_{V}\right)$ :

$$
\begin{aligned}
V_{\chi \eta} \supset & \frac{1}{\sqrt{2}}\left(\lambda_{\chi \eta}^{\eta} u v_{\eta} \eta_{V} H_{\eta}+\lambda_{\chi \eta}^{\rho} u v_{\rho} \eta_{V} H_{\rho}+\lambda_{\chi \eta}^{\chi} u^{2} \eta_{V} \bar{H}+\text { h.c. }\right) \\
& +\frac{1}{\sqrt{2}}\left(\lambda_{\chi \eta}^{\eta} v_{\eta}^{2} \chi_{V} H_{\eta}+\lambda_{\chi \eta}^{\rho} v_{\eta} v_{\rho} \chi_{V} H_{\rho}+\lambda_{\chi \eta}^{\chi} u v_{\eta} \chi_{V} \bar{H}+\text { h.c. }\right)
\end{aligned}
$$

For real $\lambda^{\prime} s$, these couplings mix the Higgs sector with the real part of the V-scalars, here denoted as $\left(\chi_{V}^{r}, \eta_{V}^{r}\right)$.

The gauge-fixing Lagrangian to the complex neutral boson $V$ will arise similarly to equations (2.63) and (2.64), now with complex conjugates. Thus, on the basis $\left(\chi_{V}^{r} \eta_{V}^{r} H_{\eta} H_{\rho} \bar{H}\right)$, only the first block of $\mathbb{M}_{\xi}$ differs from zero and is equal to the result of equation (2.72):

$$
\mathbb{M}_{\xi_{V}}=\left(\begin{array}{ccc}
\xi_{V}\left(\begin{array}{cc}
u^{2} & -u v_{\eta} \\
-u v_{\eta} & v_{\eta}^{2}
\end{array}\right) & \mathbb{O} \\
\mathbb{O} & & \mathbb{O}
\end{array}\right)
$$

The contribution from $V(\chi, \rho, \eta)$ is given by

$\left.\mathbb{M}_{V}\right|_{\beta=-\frac{1}{\sqrt{3}}}=\left(\begin{array}{cc}\mathbb{M}_{\chi \eta} & \mathbb{M}_{2} \\ \mathbb{M}_{2}^{\dagger} & \mathbb{S}\end{array}\right)$ where $\quad \mathbb{M}_{2} \equiv \sqrt{2}\left(\begin{array}{lll}\lambda_{\chi \eta}^{\eta} u v_{\eta} & \lambda_{\chi \eta}^{\rho} u v_{\rho} & \lambda_{\chi \eta}^{\chi} u^{2} \\ \lambda_{\chi \eta}^{\eta} u v_{\eta} & \lambda_{\chi \eta}^{\rho} u v_{\rho} & \lambda_{\chi \eta}^{\chi} u^{2}\end{array}\right)$ 
and $\mathbb{M}_{\chi \eta}$ is defined from equation (2.69). In order to build an invertible matrix $\mathbb{D}$ for the diagonalization of $\mathbb{M}_{\xi}$, we can fill it with the rotation matrices as presented in equation (2.65) and equation (2.94). Finally, from our previous discussion, by applying a rotation for the total mass matrix $\mathbb{M}=\mathbb{M}_{\xi_{V}}+\mathbb{M}_{V}$ through $\mathbb{D}$ we obtain a block-diagonal matrix, here represented by $4 \times 4$, that can be fully diagonalized after the insertion of an additional $\overline{\mathbb{D}}$.

\subsection{Self-interactions of gauge-bosons}

The self-interactions of the gauge-bosons are mediated by

$$
\mathcal{L}_{\text {g.b. }}=-\frac{1}{4} \mathbf{W}_{\mu \nu} \cdot \mathbf{W}^{\mu \nu}-\frac{1}{4} W_{\mu \nu}^{0} W_{0}^{\mu \nu}
$$

where $\mathbf{W}_{\mu \nu}=\left(W_{\mu \nu}^{1}, W_{\mu \nu}^{2}, \cdots, W_{\mu \nu}^{8}\right)$, and

$$
W_{\mu \nu}^{a}=\partial_{\mu} W_{\nu}^{a}-\partial_{\nu} W_{\mu}^{a}+g f^{a k i} W_{\mu}^{k} W_{\nu}^{i}
$$

or, in a simplified notation,

$$
W_{\mu \nu}^{a}=\partial_{\mu} W_{\nu}^{a}-\partial_{\nu} W_{\mu}^{a}-g \operatorname{Tr}\left[\mathbb{F}^{a} \cdot \mathbb{W}_{\mu \nu}\right]
$$

with $\left(\mathbb{F}^{a}\right)_{i j}=f^{a i j}$ the structure constants, and $\left(\mathbb{W}^{\mu \nu}\right)_{i j} \equiv W_{i}^{\mu} W_{j}^{\nu}$. Before perform the sum in equation (2.132), a set of transformations to the non-diagonal fields may be applied:

$$
\begin{aligned}
& \left(\begin{array}{l}
W^{4}\left(W^{6}\right) \\
W^{5}\left(W^{7}\right)
\end{array}\right)=\frac{1}{\sqrt{2}}\left(\begin{array}{cc}
1 & 1 \\
-i & i
\end{array}\right)\left(\begin{array}{l}
V^{+q_{V}}\left(U^{+q_{U}}\right) \\
V^{-q_{V}}\left(U^{-q_{U}}\right)
\end{array}\right) \\
& \left(\begin{array}{c}
W^{1} \\
W^{2}
\end{array}\right)=\frac{1}{\sqrt{2}}\left(\begin{array}{cc}
1 & 1 \\
i & -i
\end{array}\right)\left(\begin{array}{l}
W^{+} \\
W^{-}
\end{array}\right)
\end{aligned}
$$

and, finally,

$$
\left(\begin{array}{l}
W^{0} \\
W^{3} \\
W^{8}
\end{array}\right)=\left(\begin{array}{ccc}
-s_{x} & c_{x} c_{w} & -c_{x} s_{w} \\
0 & s_{w} & c_{w} \\
c_{x} & s_{x} c_{w} & -s_{x} s_{w}
\end{array}\right)\left(\begin{array}{c}
Z^{\prime} \\
A \\
Z
\end{array}\right)
$$

One important feature of $\mathcal{L}_{\text {g.b. }}$ is the absence of $Z^{\prime}$ interactions with standard vectors at tree- and loop-level. On the other hand, the charged bosons will couple with SM at loop-level and, for illustration, we present the vertices for $V W$ and the triple $V Z A$ :

$$
\begin{aligned}
V W: \mathcal{L}_{\text {g.b. }} \supset-\frac{g^{2}}{2}\left(V_{\nu}^{-} V^{+\nu}\right. & \left.W_{\mu}^{-} W^{+\mu}+V_{\mu}^{-} W^{-\mu} V_{\nu}^{+} W^{+\nu}-2 V_{\mu}^{+} W^{-\mu} V_{\nu}^{-} W^{+\nu}\right) \\
V Z A: \mathcal{L}_{g . b .} \supset & \frac{g^{2}}{4}\left(\sqrt{3} s_{x}\left(1-2 s_{w}^{2}\right)+c_{w} s_{w}\left(1-3 s_{x}^{2}\right)\right) \\
& \times\left(V_{\nu}^{-} A^{\nu} V_{\mu}^{+} Z^{\mu}+V_{\nu}^{+} A^{\nu} V_{\mu}^{-} Z^{\mu}-2 A^{\nu} Z^{\nu} V_{\mu}^{+} V^{-\mu}\right) .
\end{aligned}
$$

Additional Feynman rules can be extracted from [1].

\section{Fermions in the $3-3-1 \mathrm{HL}$}

This section is a brief description of the fermions in the 3-3-1HL. Among four possible versions, those models with $|\beta|=\frac{1}{\sqrt{3}}$ might require a special attention. This because they introduce heavy quarks and leptons with the same electric charges as the standard particles (see equations (2.34)-(2.38)), corresponding a new pattern of mixing among different 
generations and then to a large set of original vertices at leading order [16]. We can summarize their fermion content like:

$-\beta=-\frac{1}{\sqrt{3}}:\left\{\begin{array}{l}- \text { Three additional neutral heavy leptons (or right-handed neutrinos); } \\ - \text { Two additional flavors for D quarks; } \\ - \text { One new flavor for U quarks. }\end{array}\right.$

$\cdot \beta=\frac{1}{\sqrt{3}}:\left\{\begin{array}{l}- \text { Three additional heavy leptons with the electron charge; } \\ - \text { Two additional flavors for U quarks; } \\ - \text { One new flavor for D quarks. }\end{array}\right.$

\subsection{Gauge interactions of the fermions}

The gauge interactions for fermions may be better represented in a short notation if their lefthanded triplets are written like

$$
\psi_{\alpha}^{L}=\left(\begin{array}{cc}
\mathbf{L}^{\alpha} & E^{\alpha}
\end{array}\right)_{L} ; \quad Q_{i}^{L}=\left(\begin{array}{ll}
\mathbf{q}_{i} & J_{i}
\end{array}\right)_{L} ; \quad Q_{3}^{L}=\left(\begin{array}{ll}
\mathbf{q}_{3} & J_{3}
\end{array}\right)_{L}
$$

where the index $\alpha=[e, \mu, \tau], i=[1,2]$, and the boldface is just indicating the separation between SM doublets and exotic singlets. The right-handed fields follow the usual notation for the SM, i.e. $q_{a}^{R}, l_{\alpha}^{R}$ for quarks and leptons, $J_{a}^{R}, E_{\alpha}^{R}$ for the new particles. Thus, the total gauge-kinetic Lagrangian can be written as

$$
\begin{aligned}
\mathcal{L}_{\mathrm{kin}}= & i\left\{\sum_{\alpha=e, \mu, \tau} \bar{\psi}_{\alpha}^{L} \not \supset \psi_{\alpha}^{L}+\sum_{i=1,2} \bar{Q}_{i}^{L} \not \supset Q_{i}^{L}+\bar{Q}_{3}^{L} \not \supset Q_{3}^{L}+\right. \\
& \sum_{a=1,2,3} \bar{d}_{a}^{R} \not \supset d_{a}^{R}+\sum_{a=1,2,3} \bar{u}_{a}^{R} \not \supset u_{a}^{R}+\sum_{i=1,2} \bar{J}_{i}^{R} \not \supset J_{i}^{R}+\bar{J}_{3}^{R} \not \supset J_{3}^{R} \\
& \left.\sum_{\alpha=e, \mu, \tau} \bar{l}_{\alpha}^{R} \not \supset l_{\alpha}^{R}+\sum_{\alpha=e, \mu, \tau} \bar{E}_{\alpha}^{R} \not D E_{\alpha}^{R}\right\}
\end{aligned}
$$

and each field associates its correspondent covariant derivative.

The following identity might be useful during the task of dividing the 3-3-1 interactions into SM and new physics:

$$
\left(\begin{array}{ll}
\mathbf{a}^{\dagger} & b^{*}
\end{array}\right)\left(\begin{array}{ll}
\mathbb{A} & \mathbf{x} \\
\mathbf{x}^{\dagger} & z
\end{array}\right)\left(\begin{array}{l}
\mathbf{a} \\
b
\end{array}\right)=\mathbf{a}^{\dagger} \mathbb{A} \mathbf{a}+\mathbf{a}^{\dagger} \mathbf{x} b+b^{*} \mathbf{x}^{\dagger} \mathbf{a}+b^{*} z b
$$

such that the $2 \times 2$ matrix $\mathbb{A}$ for the covariant derivative will be given by

$$
\mathbb{A}=D^{\mathrm{SM}}+\mathbf{i g}_{2 \times 2}^{Z^{\prime}}
$$

Apart from that, we define a vector of gauge bosons

$$
\mathbf{x}_{\mu} \propto \frac{g}{\sqrt{2}}\left(\begin{array}{ll}
V_{\mu} & U_{\mu}
\end{array}\right)^{\top}
$$

where the proportionality corresponds to the complex number i, for both $\mathbf{x}$ and $\mathbf{x}^{\dagger}$. The $b$ component represents the new heavy degrees of freedom, like in the field definition of equation (3.1). By applying equation (3.3) in equation (3.2) it follows 


$$
\begin{aligned}
& \mathcal{L}_{\text {kin }}=\mathrm{i}\left\{\overline{\mathbf{L}}_{\alpha}^{L} \not \mathbf{D} \mathbf{L}_{\alpha}^{L}+\overline{\mathbf{q}}_{a}^{L} \not \partial \mathbf{q}_{a}^{L}+\bar{l}_{\alpha}^{R} \not \supset l_{\alpha}^{R}+\bar{d}_{a}^{R} \not \supset d_{a}^{R}+\bar{u}_{a}^{R} \not \supset u_{a}^{R}\right\} \\
& +\mathrm{i}\left\{\overline{\mathbf{L}}_{\alpha}^{L} \mathrm{i} \mathbf{g}_{Z^{\prime}} Z^{\prime} \mathbf{L}_{\alpha}^{L}+\overline{\mathbf{L}}_{\alpha}^{L} \mathbf{x} E_{\alpha}^{L}+\bar{E}_{\alpha}^{L} \mathbf{x}^{\dagger} \mathbf{L}_{\alpha}^{L}+\bar{E}_{\alpha}^{L}\left(\not \partial+\mathrm{i} g_{Z^{\prime}} \not \mathcal{Z}+\mathrm{i} g_{Z} Z+\mathrm{i} e Q A\right) E_{\alpha}^{L}\right\} \\
& +\mathrm{i}\left\{-\overline{\mathbf{q}}_{i}^{L} i \mathbf{g}_{Z^{\prime}}^{*} \not \mathbf{Z}_{i}^{L}+\overline{\mathbf{q}}_{i}^{L} \mathbf{x}^{*} J_{i}^{L}+\bar{J}_{i}^{L} \mathbf{x}^{\top} \mathbf{q}_{i}^{L}+\bar{J}_{i}^{L}\left(\not \partial-\mathrm{i} g_{Z^{\prime}}^{*} \not Z^{\prime}-\mathrm{i} g_{Z}^{*} Z-\mathrm{i} e Q A\right) J_{\mathrm{i}}^{L}\right\} \\
& +\mathrm{i}\left\{\overline{\mathbf{q}}_{3}^{L} \mathbf{i} \mathbf{g}_{Z^{\prime}} \not Z \mathbf{q}_{3}^{L}+\overline{\mathbf{q}}_{3}^{L} \mathbf{x} J_{3}^{L}+\bar{J}_{3}^{L} \mathbf{x}^{\dagger} \mathbf{q}_{3}^{L}+\bar{J}_{3}^{L}\left(\not \partial+\mathrm{i} g_{Z^{\prime}} \not Z L+\mathrm{i} g_{Z} Z+\mathrm{i} e Q A\right) J_{3}^{L}\right\} \\
& +\mathrm{i}\left\{\bar{E}_{\alpha}^{R} \not \supset E_{\alpha}^{R}+\bar{J}_{i}^{R} \not \supset J_{i}^{R}+\bar{J}_{3}^{R} \not \supset J_{3}^{R}\right\}
\end{aligned}
$$

and note:

- The first bracket corresponds to the SM gauge kinetic piece and we have just ignored the meaningless difference in this piece due to the conjugate representation. Thus, all the indices are running through the total three generations;

- The second contains the interactions for the heavy leptons, in addition to a tree-level term with $Z^{\prime}$;

- The third contains new exotic quarks and the $Z^{\prime}$ at tree-level. After the rotation of SM fields to their mass eigenstates, the matrix components of $\mathbf{g}_{Z^{\prime}}$ give rise to flavor changing currents due to a distinct coupling with the third generation. The couplings must take into account the properties of the Gell-Mann matrices under conjugation and indices cover the first two generations;

- The fourth bracket, in the fundamental representation, is similar to the previous line;

- The last bracket contains the right-handed exotic fields. The leptonic indices cover the three generations while the following term contains the first two heavy quarks;

- The breaking of $S U(3)_{L} \otimes U(1)_{X}$ does not lead exactly to a theory invariant under $S U(2)_{L} \otimes U(1)_{Y}$, unless the mass of the vectors inside the doublet $\mathbf{x}$ are equal, valid only in the limit $u \gg v_{\eta}, v_{\rho}$.

Moreover, the next section discuss the presence of $\beta$-dependent Yukawas that mix standard and exotic fermions, thus expanding the first line into additional tree-level contributions. The equation (3.5) can be symbolically written as

$$
\mathcal{L}_{\text {kin }}=\mathcal{L}_{\text {kin }}^{S M}+\mathcal{L}_{\text {kin }}^{E_{L}}+\mathcal{L}_{\text {kin }}^{J_{\alpha L}}+\mathcal{L}_{\text {kin }}^{J_{3 L}}+\mathcal{L}_{\text {kin }}^{R}
$$

We conclude this section with a brief digression on the described vertices. The present article composes the first part of a work in progress that intends to cover the complete integration of these new heavy fields. Thus, the method will result into an effective version of the Standard Model raised through the 3-3-1 gauge structure. Here we note, for example, that the only gauge-interactions generated at leading order are those with $Z^{\prime}$. In the second part of this project we will compare the contributions of mixed terms involving exotic fields, loop-suppressed in the expansion of dimension-six operators. Thus, the complete set of terms involving $\mathbf{x}$ only contribute at this level, concealing the interactions with the non-diagonal gauge bosons. The one-loop sector of this Effective Theory will contain only the electromagnetic covariant derivative, i.e. the $U(1)$ invariant piece, apart from the interactions with $Z$. The terms with three exotic particles must be discarded. Finally, as we mentioned in the previous paragraph, for specific $\beta$-dependent interactions, the model suppression is reduced from the appearance of linear vertices on the heavy quarks. 


\subsection{Yukawa Lagrangian}

Through this last section we discuss the division of Yukawa interactions into those pieces independent of the 3-3-1HL version and those only valid for a particular value of $\beta$. In the general case, the interactions are extracted from

$$
\begin{aligned}
\mathcal{L}_{Y}= & \left(\lambda_{i, a}^{d} \bar{Q}_{L}^{i} \eta^{*} d_{R}^{a}+\lambda_{3, a}^{d} \bar{Q}_{L}^{3} \rho d_{R}^{a}+\lambda_{i, a}^{u} \bar{Q}_{L}^{i} \rho^{*} u_{R}^{a}\right. \\
& +\lambda_{3, a}^{u} \bar{Q}_{L}^{3} \eta u_{R}^{a}+\lambda_{i, k}^{j} \bar{Q}_{L}^{i} \chi^{*} J_{R}^{k}+\lambda_{3,3}^{j} \bar{Q}_{L}^{3} \chi J_{R}^{3} \\
& +\lambda_{i, a}^{d *} \bar{d}_{R}^{a} \eta^{\top} Q_{L}^{i}+\lambda_{3, a}^{d *} \bar{d}_{R}^{a} \rho^{\dagger} Q_{L}^{3}+\lambda_{i, a}^{u *} \bar{u}_{R}^{a} \rho^{\top} Q_{L}^{i} \\
& \left.+\lambda_{3, a}^{u *} \bar{u}_{R}^{a} \eta^{\dagger} Q_{L}^{3}+\lambda_{i, k}^{J *} \bar{J}_{R}^{k} \chi^{\top} Q_{L}^{i}+\lambda_{3,3}^{J *} \bar{J}_{R}^{3} \chi^{\dagger} Q_{L}^{3}\right) \\
& +\left(\lambda_{a, b}^{l} \bar{\psi}_{L}^{a} \rho l_{R}^{b}+\lambda_{a, b}^{E} \bar{\psi}_{L}^{a} \chi E_{R}^{b}+\lambda_{a, b}^{l *} \bar{l}_{R}^{a} \rho^{\dagger} \psi_{L}^{a}+\lambda_{a, b}^{E *} \bar{E}_{R}^{b} \chi^{\dagger} \psi_{L}^{a}\right),
\end{aligned}
$$

where we have tried to unify the notation presented in [2, 5] and [14]. The indices run as $a$, $b=1,2,3$ and $i, k=1,2$. On what follows we clarify the reason for changing the lepton notation.

There are a few important comments about the above $\beta$-independent Yukawa. Note that both the heavy leptons and the new exotic quarks couples only with the first breaking triplet $\chi$. If the Scalar, Vector and Kinetic Lagrangian cannot connect the high sector with the SM at leading order, the forced absence of mixing between the $\chi$ components and standard scalars may compel the new physics to emerge always by pairs of exotic fields, leaving the heavy leptons stable. One additional form to contour this feature is to consider the complete set of allowed Yukawa terms in the framework of specific versions. For the leptons:

- $\bar{\psi}_{L} \rho E_{R}$ and $\bar{\psi}_{L} \chi l_{R}$ :

The total hypercharge for these terms is $X=\frac{1}{2}-\beta \frac{\sqrt{3}}{2}$ and would be invariant for $\beta=\frac{1}{\sqrt{3}}$, i.e. in the version with a neutral $U$ gauge boson and where the heavy leptons have the same charge as the electron. The $(E e)$ mixing creates decay channels $E_{\alpha} \rightarrow S M$ via equation (3.5).

- $\bar{\psi}_{L} \eta E_{R}$ :

Since $X_{\psi}=X_{\eta}$, the total hypercharge is equal to the HL electric charge, or $X=q_{E}$, which is neutral for $\beta=-\frac{1}{\sqrt{3}}$. Apart from that, $V$ is the complex neutral gauge-boson and the $\mathrm{SM}$ portal comes from the mixing with neutrino, $(E \nu)$.

Since both triplets are in the fundamental representation, terms with conjugated scalars are forbidden for the gauge symmetry.

These additional lepton interactions appeared in the context of $|\beta|=\frac{1}{\sqrt{3}}$, where the new quarks have the same electric charges as the standard fermions. For $|\beta|=\sqrt{3}$, however, the quarks $J$ have exotic charges and cannot mix with the $U$ or $D$ type. Thus, whenever the $\beta$ dependent interactions are omitted from the potential, we may not expect new decay channels for the leptons in these type of 3-3-1 models. Since the Yukawa is the last component of our total Lagrangian, from equation (2.39) these stable particles would be electrically charged with -2 or +1 .

Similarly, new vertices can also be extracted for the quarks. The remaining $\mathcal{L}_{Y}$ components are finally classified in terms of the $\beta$ sign: 


$$
\begin{aligned}
& \text { - } \beta=+\frac{1}{\sqrt{3}} \text { : } \\
& \mathcal{L}_{Y} \supset \lambda_{i, a}^{\chi u} \bar{Q}_{L}^{i} \chi^{*} u_{R}^{a}+\lambda_{3, a}^{\chi d} \bar{Q}_{L}^{3} \chi d_{R}^{a}+\lambda_{i, k}^{\rho J} \bar{Q}_{L}^{i} \rho^{*} J_{R}^{k} \\
& +\lambda_{i, 3}^{\eta J} \bar{Q}_{L}^{i} \eta^{*} J_{R}^{3}+\lambda_{3,3}^{\rho J} \bar{Q}_{L}^{3} \rho J_{R}^{3}+\lambda_{3, i}^{\eta J} \bar{Q}_{L}^{3} \eta J_{R}^{i}+\text { h.c. } \\
& \text { - } \beta=-\frac{1}{\sqrt{3}}: \\
& \begin{aligned}
\mathcal{L}_{Y} \supset \lambda_{i, a}^{\chi d} \bar{Q}_{L}^{i} \chi^{*} d_{R}^{a}+\lambda_{3, a}^{\chi u} \bar{Q}_{L}^{3} \chi u_{R}^{a}+\lambda_{i, 3}^{\rho J} \bar{Q}_{L}^{i} \rho^{*} J_{R}^{3} \\
+\lambda_{i, k}^{\eta J} \bar{Q}_{L}^{i} \eta^{*} J_{R}^{k}+\lambda_{3, i}^{\rho J} \bar{Q}_{L}^{3} \rho J_{R}^{i}+\lambda_{3,3}^{\eta J} \bar{Q}_{L}^{3} \eta J_{R}^{3}+\text { h.c. }
\end{aligned}
\end{aligned}
$$

As before, the indices run as $a=1,2,3$ and $i, k=1,2$.

There is one important remark on the Yukawa components above-If we return to the highenergy scenario where the symmetry breaking is given exclusively by $\chi$, there will still be one generation of standard leptons and quarks that acquire mass from their mixing with the new heavy fields. In other words, the two first terms of equation (3.9), for example, is breaking the $S U(3)_{L} \otimes U(1)_{X}$ directly into $U(1)_{q}$ leaving the bottom and the top-quark massive along with $J_{i}$ and $J_{3}$. On the basis $\left(u c t J_{3}\right.$ ) this feature can be illustrated as:

$$
\left(\begin{array}{rrrr} 
& & & a_{14} \\
& \mathbb{O} & & a_{24} \\
& & & a_{34} \\
a_{14}^{*} & a_{24}^{*} & a_{34}^{*} & a_{44}
\end{array}\right) \rightarrow\left(\begin{array}{llll}
0 & & & \\
& 0 & & \\
& & m_{t} & \\
& & m_{J}
\end{array}\right) .
$$

Thus, the theory suggests that a mass hierarchy might be originated from the presence of at least two distinct breaking scales.

The splitting of $\mathcal{L}_{Y}$ into SM and new terms is almost trivial, in the sense that only the mass Lagrangian of $U$ and $D$ quarks will contribute to the standard part. Notwithstanding, the diagonalization matrices $V_{L}^{U}$ and $V_{R}^{U}$, in order to consent with our recent Higgs phenomenology, might strongly constrain the parameters of these new Higgs sector. In the universal 3-3-1HL, i.e. in the context of only $\beta$-independent processes, the exotic quarks $J_{i}$, for $i=[1$, 2], must mix through a similar pattern as the standard quarks.

\section{Conclusions}

In our brief presentation of the 3-3-1HL components, we aimed to arrange a detailed separation between Standard terms and new physics in order to prepare a model integration. In other words, we have focused on the task of select the totality of pieces that must compose a set of effective operators generated at tree-and loop-level. It has been stressed the importance of the variable $\beta$ to define a particle content and paid a special attention on an universal context defined by the $\beta$-independent vertices. Since, in principle, there is not a strong reason for a specific $3-3-1 \mathrm{HL}$ choice, we have claimed that the first step to test its original gaugestructure is to consider those processes present in all possible variants of the model. In this scenario the Yukawa sector, for example, is loop-supressed due to the exclusive presence of exotic mixed terms ${ }^{6}$.

The work intended to be complementary to the review section of [1] and considered the most general scalar self-interactions by including new $\beta$-specific allowed terms, as present in

${ }^{6}$ Namely, by vertices in pairs of new fields. 
[22]. Finally, we concluded that the omission of these particular contributions is equivalent to assume a discrete symmetry for the variant where $|\beta|=\sqrt{3}$, leaving the theory in the presence of stable particles.

\section{Acknowledgments}

The author would like to thank E R Schmitz, B L Sánchez-Vega, Javier Fuentes-Martín and A Pich for the important discussions and appreciates Vicente Pleitez for reviewing the manuscript. The work was financially supported by the National Council of Technological and Scientific Development (CNPq-Brazil) and by the Coordination for the Improvement of Higher Education Personnel (CAPES-Brazil). The author would like to express his gratitude for the hospitality of the Institute de Fsica Corpuscular (IFIC-UV) during the composition of this article.

\section{ORCID iDs}

F C Correia (10) https://orcid.org/0000-0001-7802-5118

\section{References}

[1] Cao Q-H and Zhang D-M 2016 Collider phenomenology of the 3-3-1 model arXiv:1611.09337

[2] Pisano F and Pleitez V 1992 An SU(3) x U(1) model for electroweak interactions Phys. Rev. D 46 410-7

[3] Frampton P H 1992 Chiral dilepton model and the flavor question Phys. Rev. Lett. 69 2889-91

[4] Singer M, Valle J W F and Schechter J 1980 Canonical beutral current predictions from the weak electromagnetic gauge group SU(3) X $u$ (1) Phys. Rev. D 22738

[5] Buras A J, De Fazio F, Girrbach J and Carlucci M V 2013 The anatomy of quark flavour observables in 331 models in the flavour precision era J. High Energy Phys. JHEP02(2013)023

[6] Buras A J and De Fazio F $2016 \varepsilon^{\prime} / \varepsilon$ in 331 models J. High Energy Phys. JHEP03(2016)010

[7] Buras A J and De Fazio F 2016331 models facing the tensions in $\Delta F=2$ processes with the impact on $\varepsilon^{\prime} / \varepsilon, B_{s} \rightarrow \mu^{+} \mu^{-}$and $B \rightarrow K^{*} \mu^{+} \mu^{-} J$. High Energy Phys. JHEP08(2016)115

[8] De Conto G and Pleitez V 2016 Neutron electric dipole moment in the minimal 3-3-1 model arXiv: 1606.01747

[9] Kelso C, Long H N, Martinez R and Queiroz F S 2014 Connection of g -2 , electroweak, dark matter, and collider constraints on 331 models Phys. Rev. D 90113011

[10] Mizukoshi J K, de S Pires C A, Queiroz F S and Rodrigues da Silva P S 2011 WIMPs in a 3-3-1 model with heavy Sterile neutrinos Phys. Rev. D 83065024

[11] Profumo S and Queiroz F S 2014 Constraining the $Z^{\prime}$ mass in 331 models using direct dark matter detection Eur. Phys. J. C 742960

[12] De Conto G, Machado A C B and Pleitez V 2015 Minimal 3-3-1 model with a spectator sextet Phys. Rev. D 92075031

[13] Buras A J, Fazio F D and Girrbach J 2014331 models facing new $b \rightarrow s \mu^{+} \mu^{-}$data J. High Energy Phys. JHEP02(2014)112

[14] Fonseca R M and Hirsch M 2016 Lepton number violation in 331 models Phys. Rev. D 94115003

[15] Pleitez V and Tonasse M D 1993 Heavy charged leptons in an SU(3)-L x U(1)-N model Phys. Rev. D 48 2353-5

[16] Pleitez V 1996 New fermions and a vector-like third generation in SU(3) (C) x SU(3) (L) x U(1) (N) models Phys. Rev. D 53 514-26

[17] Ozer M 1996 SU(3)-L x U(1)-x model of the electroweak interactions without exotic quarks Phys. Rev. D 54 1143-9

[18] Diaz R A, Martinez R and Ochoa F 2004 The scalar sector of the SU(3)(c) x SU(3)(L) x U(1)(X) model Phys. Rev. D 69095009 
[19] Diaz R A, Martinez R and Ochoa F 2005 SU(3)(c) x SU(3)(L) x U(1)(X) models for beta arbitrary and families with mirror fermions Phys. Rev. D 72035018

[20] Branco G, Lavoura L and Silva J 1999 CP Violation (Oxford: Clarendon)

[21] Frampton P H 2003 Strong electroweak unification at about 4-TeV Mod. Phys. Lett. A 18 1377-82

[22] Sánchez-Vega B L, Schmitz E R and Montero J C 2016 New constraints on the 3-3-1 model with right-handed neutrinos arxiv:1612.03827 